3 Xiaodong Liu ${ }^{1,2,3,19}$, John F. Ouyang ${ }^{4,19}$, Fernando J. Rossello ${ }^{1,2,3,16,19}$, Jia Ping Tan ${ }^{1,2,3}$, 4 Kathryn C. Davidson ${ }^{1,2,3}$, Daniela S. Valdes ${ }^{1,2,3}$, Jan Schröder ${ }^{1,2,3}$, Yu B.Y. Sun ${ }^{1,2,3}$, Joseph 5 Chen $^{1,2,3}$, Anja S. Knaupp ${ }^{1,2,3}$, Guizhi Sun ${ }^{1,2,3}$, Hun S. Chy ${ }^{3,5}$, Ziyi Huang ${ }^{3,5}$, Jahnvi 6 Pflueger $^{6,7}$, Jaber Firas ${ }^{1,2,3}$, Vincent Tano ${ }^{1,2,3}$, Sam Buckberry ${ }^{6,7}$, Jacob M. Paynter ${ }^{1,2,3}$, 7 Michael R. Larcombe $e^{1,2,3}$, Daniel Poppe ${ }^{6,7}$, Xin Yi Choo ${ }^{1,2,3}$, Carmel M. O’Brien ${ }^{3,5}$, William 8 A. Pastor ${ }^{8,11,17}$, Di Chen ${ }^{8,11}$, Anna L. Leichter ${ }^{12}$, Haroon Naeem ${ }^{13}$, Pratibha Tripathi ${ }^{1,2}$, Partha 9 P. Das ${ }^{1,2}$, Alexandra Grubman ${ }^{1,2,3}$, David R. Powell ${ }^{13}$, Andrew L. Laslett ${ }^{3,5}$, Laurent 10

\section{Human reprogramming roadmap unveils route to induced trophoblast stem cells}

David $^{14,15}$, Susan K. Nilsson ${ }^{3,5}$, Amander T. Clark ${ }^{8,9,10,11}$, Ryan Lister ${ }^{6,7}$, Christian M.

Nefzger ${ }^{1,2,3,18}$, Luciano G. Martelotto ${ }^{12}$, Owen J. L. Rackham ${ }^{4}$ and Jose M. Polo ${ }^{1,2,3 *}$

${ }^{1}$ Department of Anatomy and Developmental Biology, Monash University, Victoria, Australia

${ }^{2}$ Development and Stem Cells Program, Monash Biomedicine Discovery Institute, Victoria, Australia

${ }^{3}$ Australian Regenerative Medicine Institute, Monash University, Victoria, Australia

${ }^{4}$ Program in Cardiovascular and Metabolic Disorders, Duke-National University of Singapore Medical School, Singapore

${ }^{5}$ Biomedical Manufacturing, Commonwealth Scientific and Industrial Research Organisation, Victoria, Australia

${ }^{6}$ Australian Research Council Centre of Excellence in Plant Energy Biology, School of Molecular Sciences, The University of Western Australia, Western Australia, Australia

${ }^{7}$ The Harry Perkins Institute of Medical Research, Western Australia, Australia

${ }^{8}$ Department of Molecular Cell and Developmental Biology, University of California Los Angeles, CA, USA

${ }^{9}$ Molecular Biology Institute, University of California Los Angeles, CA, USA

${ }^{10}$ Jonsson Comprehensive Cancer Center, University of California Los Angeles, CA, USA

${ }^{11}$ Eli and Edythe Broad Center of Regenerative Medicine and Stem Cell Research, University of California Los Angeles, CA, USA 
$31{ }^{12}$ Single Cell Innovation Laboratory, University of Melbourne Centre For Cancer Research,

32 The University of Melbourne, Victoria, Australia

$33 \quad{ }^{13}$ Monash Bioinformatics Platform, Monash University, Victoria, Australia

${ }^{14}$ Nantes Université, CHU Nantes, Inserm, CRTI, UMR 1064, ITUN, Nantes, France.

${ }^{15}$ Nantes Université, CHU Nantes, Inserm, CNRS, SFR Santé, FED 4203, Inserm UMS 016, 36 CNRS UMS 3556, Nantes, France.

37 Present address: ${ }^{16}$ University of Melbourne Centre For Cancer Research, The University of 38 Melbourne, Victoria, Australia

39 Present address: ${ }^{17}$ Department of Biochemistry, McGill University, Montreal, Canada

40 Present address: ${ }^{18}$ Institute for Molecular Bioscience, University of Queensland, Queensland,

41 Australia

$42 \quad{ }^{19}$ These authors contributed equally

*Correspondence: owen.rackham@duke-nus.edu.sg (O.J.L.R.); jose.polo@monash.edu (J.M.Polo)

\section{Summary Paragraph}

Reprogramming human somatic cells to primed or naive induced pluripotent stem cells (iPSC) recapitulates the different stages of early human embryonic development ${ }^{1-6}$. The molecular mechanism underpinning the reprogramming of human somatic cells to primed or naive induced pluripotency remains largely unexplored, impeding our understanding and limiting rational improvements to reprogramming protocols. To address this, we reconstructed molecular reprogramming trajectories using single-cell transcriptomics. This revealed that reprogramming into primed and naive human pluripotency follows diverging and distinct trajectories. Moreover, genome-wide accessible chromatin analyses showed key changes in regulatory elements of core pluripotency genes, and orchestrated global changes in chromatin accessibility over time. Integrated analysis of these datasets unveiled an unexpected role of trophectoderm (TE) lineage-associated transcription factors and the existence of a subpopulation of cells that enter a TE-like state during reprogramming. Furthermore, this TE-like state could be captured, allowing the derivation of induced

61 Trophoblast Stem Cells (iTSCs). iTSCs are molecularly and functionally similar to TSCs derived from human blastocysts or first-trimester placental trophoblasts ${ }^{7}$. Altogether, these 
64 reprogramming, revealing an unanticipated role of the TE-lineage specific regulatory

65 program during this process and facilitating the direct reprogramming of somatic cells into 66 iTSCs.

\section{Keywords}

68 Naive human induced pluripotent stem cells, Primed human induced pluripotent stem cells, 69 Reprogramming, Induced trophoblast stem cells, Syncytiotrophoblast, Extravillous trophoblast, Yamanaka factors, Non-integrating, Epiblast, Trophectoderm, Pre-implantation.

\section{Main Text}

73 Human embryonic stem cells (hESCs) are derived from the epiblast of preimplantation 74 blastocysts. Alternatively, human induced pluripotent stem cells (hiPSCs) are generated from 75 adult cells, such as fibroblasts, by transcription factor (TF)-mediated nuclear reprogramming. Both cell types are pluripotent since they can give rise to all cell types within the embryo, but not the extraembryonic tissues (i.e. placenta). Conventionally, hESCs/hiPSCs are cultured in the primed state resembling the post-implantation epiblast, however recently culture conditions have enabled the generation of naive hESCs/hiPSCs, resembling human preimplantation epiblast, an earlier stage in embryonic development ${ }^{1-3}$. Contrary to mouse reprogramming, where comprehensive roadmaps of the reprogramming process have been reported $^{8-12}$, few recent studies have revealed details of reprogramming towards human pluripotency ${ }^{13-15}$. Moreover, variations in donor genetic background, culture conditions, reprogramming systems and isolation strategies for reprogramming intermediates can confound results ${ }^{13-15}$.

\section{Charting a human reprogramming roadmap}

88 To investigate the cellular transitions during the reprogramming of genetically matched adult 89 human dermal fibroblasts into primed and naive hiPSCs in a clinically relevant way, we 90 utilised integration-free Sendai viruses to deliver the TFs OCT4/POU5F1, KLF4, SOX2, and $c-M Y C$ (OKSM). Transduced cells were first cultured in fibroblast medium (fm) and then transitioned into media for either primed reprogramming (pr) or t2iLGoY naive reprogramming (nr) (see Methods). Primed and naive reprogramming intermediates and hiPSCs were confirmed by morphological changes, the pluripotency marker TRA-1-60 and the naive-associated marker KLF17 (Extended Data Fig. 1a, b). To study each reprogramming pathway at single-cell resolution, we employed two complementary 
strategies: (1) 'time-resolved' to track changes happening with respect to time, by collecting intermediates at Day 0 (D0), D4, D8, D12-pr, D12-nr, D16-pr, D16-nr, D20-pr, D20-nr, D24pr, D24-nr, Passage 3 (P3-nr), P20-pr, P20-nr and subjecting them to single-nucleus RNA sequencing (snRNA-seq) (Fig. 1a); (2) 'media-resolved' to assess the entire reprogramming experiment as a single process and control for any possible confounding effects, by pooling the complete trajectories into three libraries based on the medium compositions (libraries FM, $\mathrm{PR}$, and NR) and subjecting them to single-cell RNA sequencing (scRNA-seq) (Extended Data Fig. 1c). We integrated the sn and scRNA-seq datasets, resulting in a dataset of 43,791 cells, robustly detecting 11,549 genes (Extended Data Fig. 1d, Supplementary Table 1,2, see Methods). To visualize the relationships between single cells, we employed force-directed layout $(\mathrm{FDL})^{16}$, previously used to characterise mouse reprogramming ${ }^{12}$. FDL shows that cells separated into either primed or naive reprogramming trajectories (Fig. 1b, Extended Data Fig. 1e-i, Supplementary Video 1) and identified cells in different predicted stages of the cell cycle (Extended Data Fig. 1h). Cell identity was further confirmed by the expression of known marker genes for fibroblasts (ANPEP), shared pluripotency (NANOG), primed pluripotency (ZIC2), and naive pluripotency (DNMT3L) (Fig. 1c,d, Extended Data Fig. 1j). We further corroborated these findings by applying several complementary dimensionality reduction methods such as principal component analysis (PCA), diffusion maps ${ }^{17}$ (DM), and UMAP, which produced equivalent results (Extended Data Fig. 1k-r). CytoTRACE ${ }^{18}$, which estimates cell potency, resolved the expected order with naive cells appearing the least differentiated, followed by primed and then fibroblasts (Fig. 1e). Furthermore, a pseudotime trajectory analysis using the Monocle $3^{19}$ algorithm reinforced the observed major bifurcations that occur between naive and primed trajectories, fibroblasts, and refractory cells (Fig. 1e). Altogether, these results show the naive reprogramming trajectory is distinct from the primed, rather than an extension of it.

\section{Alternative induced pluripotent conditions}

To further characterise the cell populations arising during reprogramming, we performed unsupervised clustering analysis ${ }^{20}$, identifying 21 cell clusters (Extended Data Fig. 2a). Notably, we only observed Naive Reprogramming (NR) and Primed Reprogramming (PR) intermediates near the trajectories bifurcation point. The clusters allowed us to apply Partition-based graph abstraction (PAGA) ${ }^{21}$ trajectory inference, which confirmed that PR and NR trajectories bifurcate (Extended Data Fig. 2b-d, Fig. 1f). Furthermore, the mesenchymal-epithelial transition (MET) occurred early during reprogramming (Extended 
131 Data Fig. 2e). We performed a differential gene expression analysis to identify cluster-

132 specific marker genes, which were then combined to produce eight different gene signatures 133 (Extended Data Fig. 2f-h, Supplementary Table 3), with two of these robustly distinguishing 134 primed and naive human hiPSCs. Consistent with a previous study ${ }^{14}$, we found that some 135 cells during PR activated the naive signatures, but these cells are still transcriptionally distinct 136 from naive reprogramming intermediates (Extended Data Fig. 2g, Fig. 1g). Furthermore, the 137 results demonstrated that reprogramming into naive pluripotency does not require a transition 138 through a primed pluripotency state.

140 Analysis of the gene expression of pluripotency-associated cell surface markers ${ }^{22}$ across 141 clusters informed a flow cytometry isolation strategy to analyse purified populations of 142 reprogramming intermediates using bulk-level assays (Extended Data Fig. 3a, Supplementary 143 Fig. 1, see Methods). Bulk RNA-seq obtained from different time points during primed and 144 naive reprogramming confirmed our isolation strategy (Extended Data Fig. 3b). The 145 development of different culture conditions to propagate and maintain naive hESCs/hiPSCs 146 has been a subject of active research ${ }^{1-6}$, with different media producing hiPSCs with a 147 spectrum of naive characteristics ${ }^{4}$. Thus, to study the reprogramming pathways in different 148 media conditions we isolated reprogramming intermediates in other naive media including 149 5iLAF $^{2}, \mathrm{NHSM}^{1}$, and RSeT (Extended Data Fig. 3c-e). Harmonisation of the RNA-seq of the 150 different media-intermediates with the snRNA-seq dataset revealed that NHSM cells follow 151 the previously identified primed reprogramming trajectory, whereas 5iLAF overlaps with that 152 of t2iLGoY. Day 13 and 21 intermediates of the RSeT condition transitioned along the naive 153 t2iLGoY trajectory but ultimately switched branches, establishing that RSeT hiPSCs 154 (Passage 3 and 10) clustered near primed hiPSCs (Fig. 2a, Extended Data Fig. 3f, 155 Supplementary Table 4). These observations were confirmed by scoring these 156 reprogramming intermediates using the primed and naive signatures defined previously (Fig. $1572 \mathrm{~b}$ and Supplementary Table 5). We further examined cell heterogeneity during RSeT 158 reprogramming by scRNA-seq, identifying both primed-like and naive-like intermediates 159 (Supplementary Table 6). The primed-like cell population likely dominates over time, 160 explaining the observed switch in the reprogramming branch at bulk level (Extended Data 161 Fig. 4a,b). Overall, these analyses revealed that reprogramming using various pluripotency 162 conditions always follows the main naive or primed trajectories.

\section{Chromatin dynamics during reprogramming}


165 Cell fate transitions during reprogramming are orchestrated by a dynamic reorganisation of 166 the epigenome $e^{8,10,11,14}$. To elucidate the chromatin accessibility landscape and the use of 167 regulatory elements (RE) during reprogramming, we performed Assay for Transposase168 Accessible Chromatin sequencing (ATAC-seq) on flow-cytometry-isolated reprogramming 169 intermediates (Supplementary Table 4). PCA of the ATAC-seq peaks (Fig. 2c, Extended Data 170 Fig. 5a) and its integration with RNA-seq experiments (Extended Data Fig. 5b,c, see 171 Methods) revealed distinct changes in chromatin accessibility and a bifurcated trajectory as 172 observed in our transcriptional analyses. A closer inspection of population identifying genes 173 (ANPEP, PRDM14, SOX11, DNMT3L) revealed that loss of accessibility of somatic 174 regulatory elements is accompanied by a gain of open chromatin regions in $\mathrm{RE}$ and/or 175 promoters of genes associated with either primed or naive pluripotency (Extended Data Fig. 176 5d-f). To uncover the distinct dynamics of chromatin accessibility, we performed fuzzy177 clustering ${ }^{23}$, resulting in eight clusters (C1-8) (Supplementary Table 7) and grouped them by 178 their behaviour over time (Fig. 2d). This analysis revealed: (1) Comparable distribution of 179 peaks across genomic region classes in all clusters (Extended Data Fig. 6a); (2) Regions of 180 open chromatin in fibroblasts $(\mathrm{C} 1$ and $\mathrm{C} 2$ ) became progressively inaccessible [shared loss 181 (SL)] during reprogramming, concomitant with downregulation of the associated genes (Fig. 182 2d, Extended Data Fig. 6b,c); (3) Transient clusters (C3 and C4) [shared transient (ST)] 183 exhibit overrepresentation of genes associated with transcription, metabolism, and various organ morphogenesis; (4) Regions with a gradual gain of accessibility for both primed and naive reprogramming (C5) [shared up (SU)] are associated with embryonic development and stem cell maintenance; (5) Regions that specifically gained accessibility during primed reprogramming (C6) [primed up (PU)] were associated with a range of embryonic developmental processes; (6) Two clusters (C7, C8) [naive up (NU), (C7 is also primed transient (PT)] exhibit gain of naive-specific accessibility during reprogramming and are associated with regulation of cell division, metabolism, and cell polarity (Fig. 2d, Extended Data Fig. 6b,c, Supplementary Table 8).

192

\section{Distinct programs drive reprogramming}

194 To determine specific TFs that drive these different programs, we identified TF binding-site 195 motifs enriched in each cluster (Supplementary Table 9). Motif enrichment analysis of the SL 196 regions uncovered TFs (such as FOSL1) that safeguard fibroblast cell identity, corroborating 197 previous studies in mouse ${ }^{10,11}$ (Extended Data Fig. 6d,e). C3 exhibited motifs for somatic TFs 198 (e.g. FOSL1, JUNB) and an enrichment for OCT4, SOX2, NANOG and KLF4 binding motifs 
199 (Extended Data Fig. 6d,e). This redistribution of somatic TFs to transiently accessible regions

200

201

202

203

204

205

206

207

208

209

210

211

212

213

214

215

216

217

218

219

220

221

222

223

224

225

226

227

228

229

230

231

232

harbouring their binding motifs during reprogramming by OCT4/SOX2 supports a similar effect previously described in mice ${ }^{11}$, potentially representing a pan-mammalian paradigm of somatic accessible chromatin reorganization mediated by reprogramming factors. Interestingly, two clusters (C7 and C8) show an unexpected significant motif enrichment of trophectoderm (TE) associated TFs (e.g. TFAP2C, GATA2), and these TFs were specifically upregulated during reprogramming to the naive state or transiently upregulated in the primed state (e.g. C7) (Extended Data Fig. 6d-f, Fig. 2e). Furthermore, the shared C5 cluster also exhibited enrichment for the same factors (Fig. 2e). To test whether these TE-associated TFs were passengers or drivers, we experimentally knocked them down during reprogramming using short hairpin (sh) RNAs (Extended Data Fig. 6g, Supplementary Table 10). While the absence of TFAP2C showed a minor effect on the efficiency of primed reprogramming, naive reprogramming was greatly impaired (Fig. 2f). Knockdown (KD) of GATA2 affected both primed and naive reprogramming, possibly being a result of GATA2 expression being upregulated earlier in reprogramming (Fig. 2f). Thus, these different transcriptional regulatory processes likely govern naive and primed branches of reprogramming.

\section{Trophectoderm branch during reprogramming}

We hypothesized that TE-lineage associated regulatory networks synergistically govern the transition to naive pluripotency. Thus, using our defined signatures we calculated a primed and naive score of in vivo human embryo datasets from two studies ${ }^{24,25}$ (Extended Data Fig. 7a,b, see Methods). As expected, epiblast (EPI) scored the highest for naive (Supplementary Table 11), validating our approach. We next used EPI, primitive endoderm (PE), and TE signatures (Supplementary Table 12) from a published scRNA-seq human embryo dataset ${ }^{25}$ to compute the EPI, PE, and TE scores of our reprogramming intermediates. In addition to the expected upregulation and maintenance of the EPI-associated transcriptional circuitry, TE-associated transcriptional programs were transiently activated during reprogramming into the naive t2iLGoY and 5iLAF states (Extended Data Fig. 7c-f). This was supported by a gene set enrichment analysis (Extended Data Fig. 7e). Interestingly, we found a subpopulation of cells highly enriched for the TE signatures in the single-cell trajectory of naive reprogramming (Fig. 3a, Extended Data Fig. 7g). This subpopulation forms a novel intermediates cluster (nic) and its corresponding signature (novel-intermediates signature) shows high enrichment in the TE-lineage of in vivo human blastocysts (Extended Data Fig. $7 \mathrm{~h})$. 


\section{Deriving induced trophoblast stem cells}

235 We hypothesised that this TE-associated cell cluster could be stabilised to give rise to 236 trophoblast stem cells (TSCs). Thus, we transitioned naive reprogramming intermediates at 237 day $21(\mathrm{~d} 21 \mathrm{n})$ into the recently reported human TSC medium ${ }^{7}$ (Fig. 3b). Remarkably, we 238 observed the appearance of cells that morphologically resemble TSCs, which we named 239 induced TSC (iTSC ${ }^{\mathrm{d} 21 \mathrm{n}}$ ) (Fig. 3c). Further characterization showed that iTSC ${ }^{\mathrm{d} 21 \mathrm{n}}$ express key 240 markers that define human TE and TSCs ${ }^{7,26}$ such as P63, TFAP2C, GATA2, and KRT7 (Fig.

241 3d, Extended Data Fig. 8a). Moreover, these iTSCs express comparable levels of TSC marker 242 genes and are distinct from human fibroblasts and primed and naive hiPSCs (Extended Data 243 Fig. 8b). To functionally characterize the iTSC $^{\mathrm{d} 21 \mathrm{n}}$, we examined their in vitro differentiation 244 capacity to give rise to syncytiotrophoblast (ST) and extravillous trophoblast (EVT) cells, the 245 major trophoblast subtypes of the placenta ${ }^{26}$. This demonstrated that iTSC ${ }^{\mathrm{d} 21 \mathrm{n}}$ can be 246 differentiated into ST cells characterised by SDC1-positive multinucleated cells and EVT 247 cells defined by upregulation of HLA-G, a key histocompatibility molecule expressed in 248 placenta $^{7,26}$ (Fig. 3e, Extended Data Fig. 8c). The iTSC ${ }^{\mathrm{d} 21 \mathrm{n}}$-ST cells showed significantly 249 higher fusion index compared to iTSC ${ }^{\mathrm{d} 2 \mathrm{n}}$ and secreted human chorionic gonadotropin (hCG) 250 that could be detected using an over-the-counter (OTC) human hCG pregnancy test stick and 251 quantified by hCG ELISA (Extended Data Fig. 8d-f). Next, we evaluated the in vivo 252 differentiation potential of $\mathrm{iTSC}^{\mathrm{d} 21 \mathrm{n}}$ by subcutaneous injection into mice. Nine days post253 injection (P.I.), mouse urine was positive for hCG using the OTC human pregnancy tests 254 (Fig. 3f, see Methods) and hCG was also detected in the blood serum (Extended Data Fig. $2558 \mathrm{~g})$. We further confirmed engraftment and differentiation by histology analyses of the 256 lesions formed, showing SDC1-positive ST-like cells and HLA-G-positive EVT-like cells 257 comparable to the reported primary tissue-derived TSCs ${ }^{7}$ (Extended Data Fig. 8h,i, Fig. 3g). 258 Importantly, these results demonstrate that iTSCs ${ }^{\mathrm{d} 2 \mathrm{n}}$ are bipotent in vitro and in vivo. Finally, 259 we used CD70-low to enrich TE-like cells from the 'nic' cluster and demonstrated that the 260 identified TE-like cluster carries the greatest potential for iTSC $^{\mathrm{d} 21 \mathrm{n}}$ generation (Extended 261 Data Fig. 8j,k). Altogether, this suggests that cell fate specification is highly dynamic and 262 plastic during human somatic cell reprogramming.

\section{Reprogramming fibroblasts directly into iTSCs}

265 To test whether iTSCs could be derived directly from human fibroblasts, we started 266 reprogramming experiments and transitioned the day 8 intermediates into (1) TSC or (2) 
naive medium, or (3) kept them in fibroblast medium. We then performed scRNA-seq on these conditions at day 21 to assess the cellular heterogeneity (Extended Data Fig. 9a). A population of TE-like cells was observed, and closer examination revealed that this TE-like population contained cells from all three reprogramming conditions (Fig. 4a,b, Extended Data 271 Fig. 9b-d, Supplementary Table 13). Furthermore, the day 21 fibroblast intermediates also 272 consist of cells with strong epiblast, primed, and naive signatures (Extended Data Fig. 9e), 273 and accordingly they were able to give rise to pluripotent and trophoblast stem cell lines 274 (Extended Data Fig. 9f-h). We noticed that the proportion of TE-like population was the 275 highest in TSC media compared to fibroblast and naive media (Fig. 4b, Extended Data Fig. 9d). Therefore, we hypothesized we could derive iTSC lines more efficiently by directly transitioning day 8 intermediates into TSC media $\left(\mathrm{iTSC}^{\mathrm{d} 8}\right)$, without the need to expose the cells to naive medium or prolonged culturing in fibroblast medium (Fig. 4c). As seen in Fig. $4 \mathrm{~d}, \mathrm{iTSCs}^{\mathrm{d} 8}$ were successfully derived directly, and our transgene-free iTSCs ${ }^{\mathrm{d} 8}$ (Extended Data Fig. 10a) have demonstrated the capacity to undergo $>50$ passages thus far without a 281 growth rate reduction. We then performed a comprehensive molecular and functional characterisation of $\mathrm{iTSC}^{\mathrm{d} 8}$ based on features defined for TSCs generated from primary sources ${ }^{7,26-29}$. This demonstrated that: (1) These iTSC $^{\mathrm{d} 8}$ expressed key marker genes indicative of mononuclear trophoblasts ${ }^{26}$ (Fig. 4e), and (2) they could differentiate into STs and EVTs. The STs expressed SDC1, displayed cell fusion and hCG secretion (Fig. 4f-g, Extended Data Fig. 10b-e). EVTs expressed HLA-A, B, C pan markers, but not HLA-B marker, and importantly they did express HLA-G (Extended Data Fig. 10f-h). We found that the expression of HLA-A, B, C was detected in iTSCs, similar to what was reported in TSCs derived from blastocysts ${ }^{7}$. In contrast, trophoblast organoids are HLA-negative ${ }^{28}$ suggesting that the culture conditions might support TSCs at different stages of gestation. (3) Furthermore, our iTSCs and iTSC-derived STs/EVTs share a common transcriptomic profile with the corresponding primary cell types in other published datasets (Fig. 4h, Extended Data 293 Fig. 10i-1, Supplementary Table 14). (4) iTSCs also shows higher levels of expression of 294 microRNAs (miRNAs) from the chromosome 19 miRNA cluster (C19MC) compared to fibroblast and hiPSCs, a unique feature of primary trophoblast ${ }^{26}$ (Fig. 4i). (5) We observed specific open chromatin accessibility at the promoter and putative enhancer regions of the ELF5 locus in our iTSCs and $\mathrm{TSC}^{\mathrm{BT} 5}$ (data from ${ }^{30}$ ) (Fig. 4j), which has previously been 298 found to be hypomethylated ${ }^{7,26}$. (6) Finally, we showed that iTSC ${ }^{\mathrm{d} 8}$ could engraft into mouse tissues, differentiate into the major trophoblast-lineage cell types of the placenta in vivo, and secrete hCG in urine and serum (Fig. 4k-m, Extended Data Fig. 10m). Thus, these results 
301 confirmed that $\mathrm{iTSC}^{\mathrm{d} 8}$ derived directly from human fibroblasts are similar to the primary

302 TSCs.

303

\section{Discussion}

305 Here, we present a detailed molecular roadmap of reprogramming into primed and naive 306 human pluripotency at the single-cell level, for which we developed an interactive online tool 307 (http://hrpi.ddnetbio.com/) to facilitate easy exploration of the dataset. This roadmap revealed 308 that the two reprogramming trajectories diverge, and in order for a cell to reprogram into a 309 naive pluripotent state it does not need to first acquire a primed pluripotent state, indicating 310 that reprogramming to the naive state is not a reversion of the developmental pathway. On 311 closer inspection, both the main naive and primed branches also exhibit alternative sub312 branches. We hypothesise that these sub-branches could be true alternative pathways or 313 metastable fates. For example, in the naive branch, at least two sub-branches are apparent, 314 one where a TE-associated network is upregulated and one where it is not. The fact that the 315 knockdown of TFs predicted to be driving those networks impaired naive reprogramming 316 (Fig. 2) suggests that both sub-branches are active and that the reprogramming trajectories 317 remain similar for different naive conditions (5iLAF and t2iLGoY), indicating that each 318 medium promotes not only a similar final pluripotency state, as we have shown previously ${ }^{4}$, 319 but also drives the intermediate cells along similar trajectories. Together, these results present 320 a 'push or pull' question: are similar reprogramming trajectories determined by being pulled 321 towards a common final pluripotency state, or do the specific culture media pushes the cells 322 along similar trajectories, and as a consequence result in similar final states?

323

324 The change in chromatin accessibility during primed and naive reprogramming also indicate 325 a bifurcated trajectory. Early and transient chromatin accessibility clusters are enriched in 326 OKS motifs, suggesting binding of these TFs at initially closed regions and supporting a 327 pioneering effect of these factors, as previously reported ${ }^{11,31}$. Furthermore, the upregulation 328 of TE-associated transcriptional networks during reprogramming into the epiblast-like state 329 (naive) is unexpected (Fig. 2e, 3a), since one of the first cell fate decisions that cells make 330 during development is whether they will become trophoblast or epiblast. Interestingly, our 331 results revealed the coexistence of primed-like, naive-like, and TE-like cells during 332 reprogramming in the fibroblast medium, without exposing them to any pluripotent or 333 trophoblast media, suggesting that OKSM can induce human fibroblasts to acquire 
334 pluripotent and trophoblast states. The direct reprogramming of fibroblasts into iTSCs is in 335 contrast to the recently reported three-step-approach where somatic cells must first be 336 reprogrammed into hiPSCs, then converted into the expanded-potential or naive stem cells 337 before being differentiated into $\mathrm{TSCs}^{30,32}$. We envision that this direct approach will facilitate 338 the generation of patient-specific iTSCs to study trophoblast dysfunction. Such studies are 339 critically needed as this dysfunction leads to various complications during pregnancy, such as 340 preeclampsia and intrauterine growth restriction ${ }^{7,26,28}$. Furthermore, having stable, self341 renewing, bona fide isogenic human iPSC and iTSC lines will provide a unique opportunity 342 to study human trophectoderm and trophoblast development and to better understand their 343 roles in coordinating events associated with cell fate decisions during early human 344 embryogenesis. As such, it would be possible to investigate the interaction between 345 pluripotent and trophoblast stem cells in vitro and apply modern biochemical and molecular 346 techniques at scale, rapidly increasing our ability to understand and intervene in 347 developmental diseases. Finally, since both embryonic and extraembryonic lineages can be 348 derived, these results also hint at the intriguing possibility that there may be a totipotent state 349 during reprogramming. Thus if the conditions to stabilize these cells and stringently defined 350 totipotency criteria are $\operatorname{met}^{33}$, a totipotent cell type could eventually be derived by 351 reprogramming.

\section{References}

354 1. Gafni, O. et al. Derivation of novel human ground state naive pluripotent stem cells.

$355 \quad$ Nature 504, 282-286 (2013).

356 2. Theunissen, T. W. et al. Systematic Identification of Culture Conditions for Induction 357 and Maintenance of Naive Human Pluripotency. Cell Stem Cell 15, 524-526 (2014).

358 3. Takashima, Y. et al. Resetting Transcription Factor Control Circuitry toward Ground359 State Pluripotency in Human. Cell 162, 452-453 (2015).

4. Liu, X. et al. Comprehensive characterization of distinct states of human naive pluripotency generated by reprogramming. Nat. Methods 14, 1055-1062 (2017).

362 5. Kilens, S. et al. Parallel derivation of isogenic human primed and naive induced pluripotent stem cells. Nat. Commun. 9, 360 (2018). 
364 6. Giulitti, S. et al. Direct generation of human naive induced pluripotent stem cells from

365 somatic cells in microfluidics. Nat. Cell Biol. 21, 275-286 (2019).

366 7. Okae, H. et al. Derivation of Human Trophoblast Stem Cells. Cell Stem Cell 22, 50-

$367 \quad 63 . \mathrm{e} 6(2018)$.

368 8. Polo, J. M. et al. A molecular roadmap of reprogramming somatic cells into iPS cells.

$369 \quad$ Cell 151, 1617-1632 (2012).

370 9. O’Malley, J. et al. High-resolution analysis with novel cell-surface markers identifies

371 routes to iPS cells. Nature 499, 88-91 (2013).

372 10. Chronis, C. et al. Cooperative Binding of Transcription Factors Orchestrates

373 Reprogramming. Cell 168, 442-459.e20 (2017).

374 11. Knaupp, A. S. et al. Transient and Permanent Reconfiguration of Chromatin and

375 Transcription Factor Occupancy Drive Reprogramming. Cell Stem Cell 21, 834-845.e6

$376 \quad$ (2017).

377 12. Schiebinger, G. et al. Optimal-Transport Analysis of Single-Cell Gene Expression

378 Identifies Developmental Trajectories in Reprogramming. Cell 176, 1517 (2019).

379 13. Takahashi, K. et al. Induction of pluripotency in human somatic cells via a transient

380 state resembling primitive streak-like mesendoderm. Nat. Commun. 5, 3678 (2014).

381 14. Cacchiarelli, D. et al. Integrative Analyses of Human Reprogramming Reveal Dynamic

$382 \quad$ Nature of Induced Pluripotency. Cell 162, 412-424 (2015).

383 15. Wang, Y. et al. Unique molecular events during reprogramming of human somatic cells

384 to induced pluripotent stem cells (iPSCs) at naïve state. Elife 7, (2018).

385 16. Jacomy, M., Venturini, T., Heymann, S. \& Bastian, M. ForceAtlas2, a continuous graph

386 layout algorithm for handy network visualization designed for the Gephi software. PLoS

$387 \quad$ One 9, e98679 (2014).

388 17. Haghverdi, L., Buettner, F. \& Theis, F. J. Diffusion maps for high-dimensional single- 
cell analysis of differentiation data. Bioinformatics 31, 2989-2998 (2015).

18. Gulati, G. S. et al. Single-cell transcriptional diversity is a hallmark of developmental potential. Science 367, 405-411 (2020).

19. Cao, J. et al. The single-cell transcriptional landscape of mammalian organogenesis. Nature 566, 496-502 (2019).

20. Butler, A., Hoffman, P., Smibert, P., Papalexi, E. \& Satija, R. Integrating single-cell transcriptomic data across different conditions, technologies, and species. Nat. Biotechnol. 36, 411-420 (2018).

21. Wolf, F. A. et al. PAGA: graph abstraction reconciles clustering with trajectory inference through a topology preserving map of single cells. Genome Biol. 20, 59 (2019).

22. O’Brien, C. M. et al. New Monoclonal Antibodies to Defined Cell Surface Proteins on Human Pluripotent Stem Cells. Stem Cells 35, 626-640 (2017).

23. Kumar, L. \& E Futschik, M. Mfuzz: a software package for soft clustering of microarray data. Bioinformation 2, 5-7 (2007).

24. Yan, L. et al. Single-cell RNA-Seq profiling of human preimplantation embryos and embryonic stem cells. Nat. Struct. Mol. Biol. 20, 1131-1139 (2013).

25. Petropoulos, S. et al. Single-Cell RNA-Seq Reveals Lineage and X Chromosome Dynamics in Human Preimplantation Embryos. Cell 165, 1012-1026 (2016).

26. Lee, C. Q. E. et al. What Is Trophoblast? A Combination of Criteria Define Human First-Trimester Trophoblast. Stem Cell Reports 6, 257-272 (2016).

27. Vento-Tormo, R. et al. Single-cell reconstruction of the early maternal-fetal interface in humans. Nature 563, 347-353 (2018).

28. Turco, M. Y. et al. Trophoblast organoids as a model for maternal-fetal interactions during human placentation. Nature 564, 263-267 (2018). 
29. Haider, S. et al. Self-Renewing Trophoblast Organoids Recapitulate the Developmental Program of the Early Human Placenta. Stem Cell Reports 11, 537-551 (2018).

30. Dong, C. et al. Derivation of trophoblast stem cells from naïve human pluripotent stem cells. Elife 9, (2020).

31. Soufi, A. et al. Pioneer transcription factors target partial DNA motifs on nucleosomes to initiate reprogramming. Cell 161, 555-568 (2015).

32. Gao, X. et al. Establishment of porcine and human expanded potential stem cells. Nat. Cell Biol. 21, 687-699 (2019).

33. Posfai, E., Schell, J. P., Janiszewski, A., Rovic, I. \& Murray, A. Defining totipotency using criteria of increasing stringency. bioRxiv (2020).

\section{Main Fig. Legends}

Fig. 1 | Charting a human reprogramming roadmap. a, Experimental design. b, FDL of 43,791 cells, highlighting the snRNA-seq and scRNA-seq libraries. c, Expression of marker genes associated with human fibroblasts (ANPEP), shared pluripotency (NANOG). d, Naive pluripotency (DNMT3L) and primed pluripotency (ZIC2) on FDL. e, Cellular trajectory reconstruction using CytoTRACE and Monocle3. f, PAGA trajectory inference applied onto cell clusters on FDL. g, Predicted cell states using defined gene signatures on FDL. For more details on sample number and statistics, please see statistics and reproducibility section.

\section{Fig. 2 | Distinct transcriptional regulatory programs drive primed and naive human} reprogramming. a, PCA of the integrated bulk RNA-seq of primed and several types of naive reprogramming intermediates with snRNA-seq datasets (see Methods), $n \geq 2$. b, Naive and primed signatures scores of reprogramming intermediates under different conditions. c, PCA of ATAC-seq signals, $n=2$. d, Clustering analysis of ATAC-seq peaks during reprogramming. Number of peaks in each cluster is given. Solid lines and ribbons represent mean of standardized ATAC-seq signals across clusters +/- s.d. e, Motif enrichment significance (- $\log P$ value) of TFAP2C and GATA2 in ATAC-seq clusters (C1-C8). f, Reprogramming efficiency upon TFAP2C KD into primed ( $n=6$ each for control and 
443 shTFAP2C) and naive ( $n=6$ each for control and shTFAP2C) pluripotency, and 444 reprogramming efficiency upon GATA2 KD into primed ( $n=10$ for control, $n=11$ for 445 shGATA2) and naive ( $n=11$ each for control and shGATA2) pluripotency. For more details on sample number and statistics, please see statistics and reproducibility section.

447

Fig. 3 | Derivation of iTSCs during reprogramming. a, In vivo TE signatures on FDL projection overlaid with single-cell trajectories constructed using Monocle3 (black lines). Blue arrow indicates TE-enriched cell population. b, Experimental design for derivation of iTSC $^{\mathrm{d} 21 \mathrm{n}}$. c, Phase-contrast image of $\mathrm{iTSC}^{\mathrm{d} 21 \mathrm{n}}$. Scale bar, $100 \mu \mathrm{m}$. d, Immunostaining of iTSC $^{\mathrm{d} 21 \mathrm{n}}$ with P63, TFAP2C, GATA2, KRT7. Scale bar, $100 \mu \mathrm{m}$. Representative images from $n=4$. e, SDC1 and HLA-G immunostaining of ST and EVT cells, respectively, differentiated from iTSC ${ }^{\mathrm{d} 21 \mathrm{n}}$. Scale bar, $100 \mu \mathrm{m}$. Representative images from $n=4$. f, Representation of iTSC $^{\mathrm{d} 21 \mathrm{n}}$ engraftment assay by injection into NOD-SCID mice. The urine, blood serum, and lesions were examined 9 days post-injection. Representative positive results for hCG pregnancy test from urine samples collected from iTSC ${ }^{\mathrm{d} 2 \mathrm{ln}}$-injected mice compared to the vehicle controls, $n=3$. g, Immunohistochemical staining of SDC1 and HLA-G in the lesions harvested from iTSC ${ }^{\mathrm{d} 21 \mathrm{n}}$-engrafts in NOD-SCID mice. No evident lesions were observed in vehicle controls. Scale bar, $200 \mu \mathrm{m}$. Representative images from $n=4$. For more details on sample number and statistics, please see statistics and reproducibility section.

462

463

Fig. 4 | Direct derivation of iTSCs from human fibroblasts. a, FDL representation of 464 scRNA-seq libraries of day 21 reprogramming intermediates (10,518 cells). b, TE signatures on FDL projections, TE-like population is highlighted and coloured by the library. c, 466 Experimental design of direct derivation of $\operatorname{iTSC}^{\mathrm{d} 8}$ from fibroblasts. d, Phase-contrast image 467 of $\mathrm{iTSC}^{\mathrm{d} 8}$. Scale bar, $100 \mu \mathrm{m}$. e, Immunostaining of $\mathrm{iTSC}^{\mathrm{d} 8}$ for several TSC makers. Scale bar, $100 \mu \mathrm{m}$. f, Phase-contrast and immunostaining of ST and g, EVT cells differentiated from iTSC $^{\mathrm{d} 8}$. Scale bar, $100 \mu \mathrm{m}$. $n=4$ for $\mathbf{d - g}$. h, Spearman correlation of transcriptomes from this study with published datasets. Biological replicates $(n \geq 2)$ are averaged prior to performing correlation. i, C19MC miRNAs expression normalised to miR-103a, mean \pm s.e.m., not detected (ND), red dotted line indicates level in primed hiPSCs. $n=2$. j, ATAC-seq signal at ELF5 region in indicated cell types $\left(\mathrm{TSC}^{\mathrm{BT} 5}\right.$ derived from human blastocysts ${ }^{30}$ ), mean value of replicates $(n=2)$, TSC peaks are marked in grey. $\mathbf{k}$, Representative hCG test from urine samples collected from iTSC $^{\mathrm{d} 8}$-injected mice, $n=3$. $\mathbf{l}$, hCG protein level detected by hCG 476 ELISA using mouse blood serum samples, $n=4$. m, Hematoxylin and eosin, and 
477 immunohistochemical staining of KRT7, SDC1 and HLA-G in the lesions harvested from

478 iTSC $^{\mathrm{d} 8}$-engrafts in NOD-SCID mice, $n=4$, no evident lesions were observed in vehicle 479 controls. Scale bar, $200 \mu \mathrm{m}$. For more details on sample number and statistics, please see 480 statistics and reproducibility section.

481

482

\section{METHODS}

Cell culture conditions. The experimental design, materials, and reagents are described in 484 the Life Sciences Reporting Summary. All cell lines used in this study were authenticated, mycoplasma tested as described in the Reporting Summary. Primary human adult dermal fibroblasts $(\mathrm{HDFa})$ from three different female donors were obtained from ThermoFisher (Catalogue number C-013-5C and lot\#1029000 for 38F, lot\#1528526 for 55F and lot\#1569390 for 32F), cells were recovered and plated in medium 106 (ThermoFisher) supplemented with low serum growth supplement (LSGS) (ThermoFisher) for expansion. The use of human embryonic stem cells (H9) was carried out in accordance with approvals from Monash University and the Commonwealth Scientific and Industrial Research Organisation (CSIRO) Human Research Ethics Offices. Conventional primed human iPSCs (established lines) and H9 ESCs (WiCell Research Institute, Madison, WI, 494 http://www.wicell.org) were maintained in a feeder-free system on vitronectin (VTN-N, 495 Gibco) coated tissue culture plastics in Essential 8 medium (Gibco). Media were changed 496 daily, and cells were passaged every 5 days using $0.5 \mathrm{mM}$ EDTA (Invitrogen). Culture 497 conditions used for human somatic cell reprogramming were prepared as described 498 previously ${ }^{4,34}$. Fibroblast medium: DMEM (ThermoFisher), 10\% Fetal Bovine Serum (FBS, 499 Hyclone), 1\% Nonessential amino acids (ThermoFisher), 1mM GlutaMAX (ThermoFisher), $5001 \%$ Penicillin-streptomycin (ThermoFisher), $55 \mu \mathrm{M}$ 2-mercaptoethanol (ThermoFisher) and $5011 \mathrm{mM}$ sodium pyruvate (ThermoFisher). Primed medium: DMEM/F12 (ThermoFisher), 20\% 502 Knockout Serum Replacement (KSR, ThermoFisher), 1mM GlutaMAX (ThermoFisher), $5030.1 \mathrm{mM}$ 2-mercaptoethanol (ThermoFisher), 1\% Non-essential amino acids (ThermoFisher), 504 50ng/mL Recombinant human FGF2 (Miltenyi Biotec), 1\% Penicillin-streptomycin 505 (ThermoFisher). Naive medium (t2iLGoY) ${ }^{35}: \quad 50: 50$ mixture of DMEM/F-12 506 (ThermoFisher) and Neurobasal medium (ThermoFisher), supplemented with 2mM L507 Glutamine (ThermoFisher), 0.1mM 2-mercaptoethanol (ThermoFisher), 0.5\% N2 supplement 508 (ThermoFisher), 1\% B27 supplement (ThermoFisher), 1\% Penicillin-streptomycin 509 (ThermoFisher), $10 \mathrm{ng} / \mathrm{ml}$ human leukemia inhibitory factor (LIF, made in house), $250 \mu \mathrm{M} \mathrm{L}-$ 
510 ascorbic acid (Sigma), 10 $\mu \mathrm{g} / \mathrm{ml}$ recombinant human insulin (Sigma), 1 $\mu \mathrm{M}$ PD0325901

511 (Miltenyi Biotec), 1 $\mu \mathrm{M}$ CHIR99021 (Miltenyi Biotec), $2.5 \mu \mathrm{M}$ Gö6983 (Tocris), 10 $\mu \mathrm{M} \mathrm{Y}$ -

51227632 (Abcam). Naive Human Stem cell Medium (NHSM): culture condition adapted from

513 Gafni and colleagues ${ }^{1}$ with suggested modifications from the Hanna laboratory's web page in

5142014 was used. DMEM/F12 (ThermoFisher) supplemented with 10mg/ml AlbuMAX I

515 (ThermoFisher), $1 \%$ Penicillin-streptomycin (ThermoFisher), 1mM GlutaMAX

516 (ThermoFisher), 1\% Nonessential amino acids (ThermoFisher), 10\% KSR (ThermoFisher),

$5175 \mathrm{ml} \mathrm{N} 2$ supplement (ThermoFisher), $12.5 \mu \mathrm{g} / \mathrm{ml}$ recombinant human insulin (Sigma),

$51850 \mu \mathrm{g} / \mathrm{ml}$ L-ascorbic acid (Sigma), 20ng/ml of recombinant human LIF (made in house),

519 8ng/ml FGF2 (Peprotech), 2ng/ml recombinant TGF- $\beta 1$ (Peprotech), 20ng/ml human LR3-

520 IGF1 (Prospec), and small molecule inhibitors: $1 \mu \mathrm{M}$ PD0325901 (Miltenyi Biotec), $3 \mu \mathrm{M}$

521 CHIR99021 (Miltenyi Biotec), 5 $\mu \mathrm{M}$ SP600125 (Tocris), $2 \mu \mathrm{M}$ BIRB796 (Axon), $0.4 \mu \mathrm{M}$

522 LDN193189 (Axon), 10 $\mu \mathrm{M}$ Y-27632 (supplemented daily to media from freshly thawed

523 stock aliquot; Abcam) and $1 \mu \mathrm{M}$ Gö6983 (supplemented daily to media from freshly thawed

524 stock aliquot; Tocris). Naive 5iLAF medium ${ }^{2,36}$ : 50:50 mixture of DMEM/F-12

525 (ThermoFisher) and Neurobasal medium (ThermoFisher) supplemented with 1\% N2

526 supplement (ThermoFisher), 2\% B27 supplement (ThermoFisher), 1\% Nonessential amino

527 acids (ThermoFisher), 1mM GlutaMAX (ThermoFisher), 1\% Penicillin-streptomycin

528 (ThermoFisher), $0.1 \mathrm{mM}$ 2-mercaptoethanol (ThermoFisher), $50 \mu \mathrm{g} / \mathrm{ml}$ Bovine Serum

529 Albumin (ThermoFisher), 1 $\mu \mathrm{M}$ PD0325901 (Miltenyi Biotec), 1 $\mu \mathrm{M}$ IM-12 (Millipore),

$530 \quad 0.5 \mu \mathrm{M}$ SB590885 (Tocris), $1 \mu \mathrm{M}$ WH-4-023 (A Chemtek), 10 $\mu \mathrm{M}$ Y-27632 (Abcam), 20ng/ml

531 Activin A (Peprotech), 8ng/ml

532 FGF2 (Miltenyi Biotec), 20ng/ml human LIF (made in house) and $0.5 \%$ KSR

533 (ThermoFisher). Naive RSeT medium: 100ml of RSeT 5X supplement, $1 \mathrm{ml}$ of RSeT 500X

534 supplement and $0.5 \mathrm{ml}$ of RSeT $1000 \mathrm{X}$ supplement into $398.5 \mathrm{ml}$ of RSeT Basal Medium;

535 (Stem Cell Technologies) supplement with 1\% Penicillin-streptomycin (ThermoFisher).

536 Human trophoblast stem cell (TSC) medium ${ }^{7}$ : DMEM/F-12, GlutaMAX (ThermoFisher)

537 supplemented with 0.3\% BSA (Sigma), 0.2\% FBS (ThermoFisher), 1\% ITS-X supplement

538 (ThermoFisher), $0.1 \mathrm{mM}$ 2-mercaptoethanol (ThermoFisher), 0.5\% Penicillin-streptomycin

539 (ThermoFisher), $1.5 \mu \mathrm{g} / \mathrm{ml}$ L-ascorbic acid (Sigma), $5 \mu \mathrm{M}$ Y27632 (Abcam), $2 \mu \mathrm{M}$

540 CHIR99021 (Miltenyi Biotec), $0.5 \mu \mathrm{M}$ A83-01 (Sigma), $1 \mu \mathrm{M}$ SB431542, $50 \mathrm{ng} / \mathrm{ml}$ EGF

541 (Peprotech) and 0.8 mM Valproic acid (VPA, Sigma). 
542 Reprogramming experiments. The naive t2iLGoY medium was used for naive

543 reprogramming as we have previously shown that it can be used to reprogram fibroblasts into

544 naive hiPSCs, with all the hallmarks of naive pluripotency and maintains a more stable 545 karyotype when compared to other conditions ${ }^{4}$. Human somatic cell reprogramming into 546 primed and naive pluripotent states experiments and subsequent culture of primed and naive 547 hiPSCs were performed as previously described ${ }^{4,34}$. Briefly, reprogramming of human 548 fibroblasts was conducted using CytoTune-iPS 2.0 Sendai reprogramming kit according to 549 the manufacturer's instructions (ThermoFisher). Primary HDFa were seeded at a density of $550 \sim 5-10 \times 10^{4}$ cells in fibroblast medium. As shown in Fig. 1a, cells were transduced with 551 Sendai viruses in FM at the multiplicity of infection (MOI) as follows, KOS MOI=5 or 10, c$552 \mathrm{MYC}$ MOI $=5$ or $10, \mathrm{KLF} 4 \mathrm{MOI}=6$ or 12 , cells were reseeded onto a layer of iMEF feeders 553 on day 7 and transitioned into different culture media (Primed, t2iLGoY, NHMS, RSeT, 554 5iLAF) the next day. After 18-21 days, hiPSCs could be passaged and expanded as described 555 previously $^{34}$. For shRNA knockdown experiments, a pair of U6 shRNA lentiviral vectors 556 (VectorBuilder) for each gene was used. The shRNA sequences are provided in 557 (Supplementary Table 10). Lentiviral particles were generated using human embryonic 558 kidney cells $(293 \mathrm{~T})$ as described previously ${ }^{11,37}$. HDFa were transduced with lentiviral 559 vectors for one week and replated two days before Sendai transduction. Colony counts of 560 TFAP2C, GATA2 knockdown experiments are provided in Source Data Fig. 2f. Knockdown 561 experiments were validated by qRT-PCR, and primers used are listed in Supplementary Table 562 15. All cells were cultured at $37{ }^{\circ} \mathrm{C}, 5 \% \mathrm{O}_{2}$ and $5 \% \mathrm{CO}_{2}$ incubators. For the derivation of $563 \mathrm{iTSC}^{\mathrm{d} 21 \mathrm{n}}$ during naive reprogramming, day 21 naive t2iLGoY reprogramming intermediates 564 were transitioned into TSC medium ${ }^{7}$. After 4-5 days, cells were passaged using TrypLE 565 express (ThermoFisher) every 3-4 days at a 1:2-1:4 ratio. For the initial 4 passages, iTSCs 566 were passaged onto iMEF feeders and cultured in a $37{ }^{\circ} \mathrm{C}, 5 \% \mathrm{O}_{2}$ and $5 \% \mathrm{CO}_{2}$ incubator. 567 Starting from passage $5, \mathrm{iTSC}^{\mathrm{d} 21 \mathrm{n}}$ were passaged onto tissue culture flask that was pre-coated 568 with $5 \mu \mathrm{g} / \mathrm{ml}$ Collagen IV (Sigma) (for at least one hour at $37{ }^{\circ} \mathrm{C}$ ) and cultured in a $37{ }^{\circ} \mathrm{C}$, $56920 \% \mathrm{O}_{2}$ and $5 \% \mathrm{CO}_{2}$ incubator. For the direct derivation of $\mathrm{iTSC}^{\mathrm{d} 8}$ from human fibroblasts, 570 day 8 fibroblast reprogramming intermediates were transitioned into TSC medium. After 1057113 days, iTSC ${ }^{\mathrm{d} 8}$ can be passaged onto iMEF feeders and cultured in a $37{ }^{\circ} \mathrm{C}, 5 \% \mathrm{O}_{2}$ and $5 \%$ $572 \mathrm{CO}_{2}$ incubator as described for iTSC ${ }^{\mathrm{d} 21 \mathrm{n}}$ above. Sendai detection in established iTSC cell 573 lines was performed as described in the Sendai reprogramming protocol (ThermoFisher). For 574 the derivation of primed, naive hiPSCs and iTSCs from d21 fibroblast reprogramming 
575 intermediates, day 21 fibroblast reprogramming intermediates were transitioned into primed,

576 naive or TSC media, and then cultured and expanded as described above.

577 Differentiation of iTSC ${ }^{\mathrm{d} 21 \mathrm{n}}$ and iTSC $^{\mathrm{d} 8}$ into ST and EVT in vitro. Differentiation of iTSCs 578 into ST and EVT was performed as previously described ${ }^{7}$. For the differentiation of iTSCs 579 into ST, iTSCs were seeded at a density of $1 \times 10^{5}$ cells per well onto a 6-well plate pre580 coated with $2.5 \mu \mathrm{g} / \mathrm{ml}$ Collagen IV (Sigma) and cultured in $2 \mathrm{ml}$ of ST differentiation 581 medium [DMEM/F-12, GlutaMAX (ThermoFisher) supplemented with 0.3\% BSA (Sigma), 582 4\% KSR (ThermoFisher), 1\% ITS-X supplement (ThermoFisher), 0.1mM 2-mercaptoethanol 583 (ThermoFisher), 0.5\% Penicillin-streptomycin (ThermoFisher), $2.5 \mu \mathrm{M}$ Y27632 (Abcam) and $5842 \mu \mathrm{M}$ forskolin (Selleckchem)]. Media were replaced daily for the initial 4 days, and cells 585 were analysed on day 6. Fusion index was used to quantify the efficiency of cell fusion, 586 which is calculated by using the number of nuclei counted in the syncytia minus the number 587 of syncytia, then divided by the total number of nuclei counted. For the differentiation of 588 iTSCs into EVT, iTSCs were seeded at a density of $0.75 \times 10^{5}$ cells per well onto a 6-well 589 plate pre-coated with $1 \mu \mathrm{g} / \mathrm{ml} \mathrm{Col} \mathrm{IV} \mathrm{(Sigma)} \mathrm{and} \mathrm{cultured} \mathrm{in} 2 \mathrm{ml}$ of EVT differentiation 590 medium [DMEM/F-12, GlutaMAX (ThermoFisher) supplemented with 0.3\% BSA (Sigma), 591 4\% KSR (ThermoFisher), 1\% ITS-X supplement (ThermoFisher), 0.1mM 2-mercaptoethanol 592 (ThermoFisher), 0.5\% Penicillin-streptomycin (ThermoFisher), $2.5 \mu \mathrm{M}$ Y27632 (Abcam), $593100 \mathrm{ng} / \mathrm{ml} \mathrm{NRG1} \mathrm{(Cell} \mathrm{Signaling)} \mathrm{and} \mathrm{7.5} \mu \mathrm{M}$ A83-01 (Sigma). Shortly after suspending the 594 cells in the EVT differentiation medium, Matrigel (Corning) was overlaid to a $2 \%$ final 595 concentration. On day 3 of differentiation, EVT differentiation medium without hNRG1 (Cell 596 Signaling) and Matrigel (Corning) was added to a final concentration of $0.5 \%$. On day 6 of 597 differentiation, EVT differentiation media were replaced without hNRG1 (Cell Signaling) or 598 KSR (ThermoFisher), and Matrigel (Corning) was added to $0.5 \%$ final concentration. The 599 cells were cultured for an additional 2 days before analyses were performed.

600 iTSC $^{\text {d21n }}$ and iTSC $^{\text {d8 }}$ in vivo engraftment assay. Protocols and use of animals were 601 undertaken with the approval of the Monash University Animal Welfare Committee 602 following the 2004 Australian Code of Practice for the Care and Use of Animals for 603 Scientific Purposes and the Victorian Prevention of Cruelty to Animals Act and Regulations 604 legislation. iTSCs with $80 \%$ confluency were dissociated with TrypLE express 605 (ThermoFisher) and counted. $10^{7}$ iTSCs were resuspended in $200 \mu$ of a 1:2 mixture of 606 Matrigel (Corning) and DMEM/F-12, GlutaMAX (ThermoFisher) supplemented with 0.3\% 
607 BSA (Sigma) and 1\% ITS-X supplement (ThermoFisher). The cellular mixture was then 608 injected subcutaneously into dorsal flanks of male and female, 5-20 weeks of age NOD/SCID 609 IL-2R Gamma KO mice (100 $\mu$ l into each flank). Mice were randomised between controls 610 and iTSCs, but not blinded. Nine days after injection, urine, blood serum, and lesions were 611 collected from the mice for analysis. Mice urine and serum were utilized for the detection and 612 measurement of hCG secretion as detailed below. Collected lesions were fixed with $4 \%$ 613 Paraformaldehyde (PFA, Sigma) overnight and subsequently embedded in paraffin. Lesions 614 collected were less than $1 \mathrm{~cm}^{3}$ in volume. Paraffin-embedded tissues were sectioned and 615 stained with hematoxylin-eosin $(\mathrm{H} \& \mathrm{E})$ or proceeded with immunohistochemistry staining of 616 KRT7, HLA-G, SDC1 (Supplementary Table 16) at the Histology Platform at Monash 617 University.

618 Pregnancy tests and hCG ELISA. iTSCs were seeded at a density of $0.5 \times 10^{5}$ cells $/ \mathrm{ml}$ on a 619 12-well plate for ST differentiation as detailed in the above section. The medium of the ST 620 cells was replaced on day 4 and the conditioned medium was collected at day 6 and stored at $621-80^{\circ} \mathrm{C}$. As controls, iTSCs were also seeded at a density of $0.5 \times 10^{5}$ cells $/ \mathrm{ml}$ on a 12 -well 622 plate and cultured in TSC medium. 2 days later, the conditioned medium was collected and 623 stored at $-80^{\circ} \mathrm{C}$. The conditioned media were then tested using OTC hCG pregnancy test 624 sticks (Freedom) according to the manufacturer's recommendations. In addition, the hCG 625 level within the media was also measured using hCG ELISA kit (Abnova, ABNOKA4005) 626 according to the manufacturer's instructions. Following the iTSC engraftment assay, the 627 collected mouse urine was tested using the OTC hCG pregnancy test sticks as described 628 above and hCG level in blood serum was measured using hCG ELISA kit as described above.

629 Flow cytometry analysis and fluorescent activated cell sorting (FACS). All antibodies 630 used in flow cytometry analysis and FACS experiments were summarized in Supplementary 631 Table 16. Cells were dissociated with TrypLE express (ThermoFisher), and DPBS 632 (ThermoFisher) supplemented with 2\% FBS (Hyclone) and 10 $\mu \mathrm{M}$ Y-27632 (Abcam) was 633 used for antibody labeling steps and final resuspension of the samples. For SPADE analysis 634 (Extended Data Fig. 3e), a three-step antibody labeling procedure was used: (1) rat anti635 human IgM SSEA-3 (1:10, BD); mouse anti-human NLGN4X IgG2a (1:128, CSIRO $636 \mathrm{CSTEM}^{22}$ ). (2) mouse anti-rat IgM PE (1:200, eBiosciences); BV605 goat anti-mouse IgG 637 (1:100, BioLegend). (3) BV421 mouse anti-human CD326 (EpCAM) (1:100, BioLegend); 638 BUV395 mouse anti-human TRA-1-60 (1:100, BD); BV711 mouse anti-human CD24 (1:50, 
639 BD); mouse anti-human SSEA-4-PE-Vio770 (1:20, Miltenyi Biotec); mouse anti-human

640 F11R IgG was conjugated to APC by the Walter and Eliza Hall Institute of Medical Research 641 (WEHI) antibody facilities (1:200, CSIRO CSTEM27 $\left.{ }^{22}\right) ;$ APC-Cy7 CD13 (1:500, 642 BioLegend); Anti-TRA-1-85 (CD147)-VioBright FITC (1:20, Miltenyi Biotec). For FACS, 643 antibody labeling was performed as below: (1) mouse anti-human F11R IgG antibody (1:200, 644 CSIRO CSTEM27); PE rat anti-human SSEA-3 IgM antibody (1:10, BD) (2) AF647 goat 645 anti-mouse $\operatorname{IgG}$ antibody (1:2,000, ThermoFisher); mouse anti-rat $\operatorname{IgM}$ PE (1:200, 646 eBiosciences). (3) PE-Cy7 mouse anti-human CD13 (1:400, BD); BV421 mouse anti-human 647 CD326 (EpCAM) (1:100, BioLegend); BUV395 mouse anti-human TRA-1-60 (1:100, BD). 648 The antibody labeling steps were carried out in a volume of $500 \mu \mathrm{l}$ per 1 million cells, and 649 incubation time was 10 mins on ice per step; after each antibody labeling step, cells were 650 washed with $10 \mathrm{ml}$ cold PBS and pelleted at $400 \times \mathrm{g}$ for 5 mins. The cells were then 651 resuspended in a final volume of $500 \mu \mathrm{l}$, and propidium iodide (PI) (Sigma) was added to a 652 concentration of $2 \mu \mathrm{g} / \mathrm{ml}$. Cell sorting was carried out with a $100 \mu \mathrm{m}$ nozzle on an Influx 653 instrument (BD Biosciences), and flow cytometry analysis was carried out using an LSRIIb 654 or LSRIIa analyser (BD Biosciences). For Supplementary Fig. 1, reprogramming 655 intermediates were isolated on day 3 into $\mathrm{CD} 13+\mathrm{F} 11 \mathrm{R}+$ and $\mathrm{CD} 13+\mathrm{F} 11 \mathrm{R}-$ subpopulations, 656 and then reseeded into FM condition for five days for flow cytometry reanalysis and for 657 hiPSC formation confirmed by alkaline phosphatase (AP) staining according to the 658 manufacturer's instructions (Vector laboratories). On day 7, CD13+, CD13-F11R+TRA-1-60659 and CD13-F11R+TRA-1-60+ subpopulations were used for such analysis (reseeded in FM 660 condition for one day and then transitioned into either primed or naive t2iLGoY conditions).

661 On day 13, CD13-F11R+TRA-1-60+SSEA3+EPCAM- and CD13-F11R+TRA-1662 60+SSEA3+EPCAM+ subpopulations were isolated for primed reprogramming, CD13663 F11R+TRA-1-60+SSEA3+EPCAM+ and CD13-F11R+TRA-1-60+SSEA3-EPCAM+ 664 subpopulations were isolated for naive reprogramming. For iTSCs purification, a two-step 665 antibody labeling procedure was used: (1) mouse anti-human APA (1:100) (2) BUV395 666 mouse anti-human TRA-1-60 (1:100, BD); APC rat anti-human \& mouse CD49F (ITGA6) 667 (1:20, Miltenyi Biotec); AF488 goat anti-mouse IgG1 antibody (1:2,000, ThermoFisher). 668 iTSCs purification was performed on the reprogrammed cells at passage 9-10 by isolating 669 TRA160-APA+ITGA6+ subpopulations and reseeding onto Col IV-coated 6-well plate for 670 long-term passaging. For Extended Data Fig. 8k, enrichment of CD70-high, CD70-low 671 populations was performed using a one-step antibody labelling procedure: anti-TRA-1-85 672 (CD147)-VioBright FITC (1:20, Miltenyi Biotec); PE-Cy7 mouse anti-human CD13 (1:400, 
673 BD); BV421 mouse anti-human CD326 (EpCAM) (1:100, BioLegend); BUV395 mouse anti674 human TRA-1-60 (1:100, BD); APC mouse anti-human F11R (1:250, CSIRO CSTEM27); 675 BUV737 mouse anti-human CD70 (1:100, BD). Details of these antibodies are provided in 676 Supplementary Table 16. Labeled cells were resuspended in a final volume of $500 \mu 1$ 677 containing $2 \mu \mathrm{g} / \mathrm{ml}$ of propidium iodide (PI) (Sigma) for cell sorting. TRA185+CD13678 F11R+TRA-1-60+EPCAM+CD70-high and TRA185+CD13-F11R+ TRA-1679 60+EPCAM+CD70-low subpopulations denoted as CD70-high and CD70-low 680 subpopulations respectively were isolated and reseeded onto a layer of iMEF feeders (24-well 681 plate) at a density of $5 \times 10^{3}$ cells per well. On the next day after reseeding, the spent culture 682 medium was replaced with the TSC medium. Immunostaining for KRT7 positive colonies 683 was then performed on day 9 after reseeding as described below. We demonstrated that the 684 CD70-low TE-like novel intermediates resulted in more KRT7+ iTSC colonies as compared 685 to unenriched or CD70-high naive populations, indicating that the identified TE-like cluster 686 carries the greatest potential for the generation of $\mathrm{iTSC}^{\mathrm{d} 21 \mathrm{n}}$ (Extended Data Fig. 8k). For HLA 687 experiments, cells were labeled with HLA-A, B, C (W6/32) or HLA-Bw4 (1:1, Purcell lab), 688 then AF647 goat anti-mouse IgG antibody (1:1000, ThermoFisher). Or cells were labeled 689 with (1) HLA-G MEM-G/9 (1:500, Abcam); (2) AF488 goat anti-mouse IgG antibody 690 (1:1000, ThermoFisher); (3) PE-Cy7 mouse anti-human HLA-A, B, C W6/32 (1:200, 691 Biolegend).

692 Multidimensional analyses of flow cytometry data. To visualise the multidimensional flow 693 cytometry data, we employed spanning-tree progression analysis of density-normalized 694 events $(\mathrm{SPADE})^{38}$. SPADE trees were generated as described previously ${ }^{39}$ using the 695 Cytobank platform (http://www.cytobank.org). Samples were labeled with antibodies as 696 described above for flow cytometry analysis and all experiments were performed on the same 697 day to warrant their use for comparison. The SPADE tree indicates a clear transition of cell 698 populations at the early stages of reprogramming (from day 0 to day 7), with reprogramming 699 in NHSM and RSeT conditions exhibiting a more primed-like transition (Extended Data Fig. 700 3e). In particular, the RSeT media formed a separated branch on the SPADE tree, in contrast 701 to reprogramming in 5iLAF and t2iLGoY (Extended Data Fig. 3e).

702 Quantitative RT-PCR. RNA was extracted from cells using RNeasy micro kit (Qiagen) or 703 RNeasy mini kit (Qiagen) and QIAcube (Qiagen) according to the manufacturer's 704 instructions. Reverse transcription was then performed using SuperScript III cDNA Synthesis 
705 Kit (ThermoFisher) or QuantiTect reverse transcription kit (Qiagen, Cat no. 205311), real-

706

707

708

709

710

711

712

713

714

715

716

717

718

719

720

721

722

723

724

725

726

727

728

729

730

731

732

733

734

735

736

time PCR reactions were set up in triplicates using QuantiFast SYBR Green PCR Kit (Qiagen) and then carried out on the 7500 Real-Time PCR system (ThermoFisher).

Quantitative RT-PCR for miRNAs. miRNA and total RNA was extracted from cells using miRNeasy Mini Kit (Qiagen, Cat no. 217004) according to the manufacturers' instructions. They were then converted to cDNA using TaqMan MicroRNA Reverse Transcription Kit (Life Technologies, Cat no. 4366596). qPCR reactions were performed using QuantiFast SYBR Green PCR Kit (Qiagen). Data obtained from miRNA qPCR was analyzed as follows: In each sample, hsa-miR-103a was used for normalization to obtain $\Delta \mathrm{Ct}$ value for each miRNA. $2^{\wedge}(-\Delta \mathrm{Ct})$ was then calculated for each miRNA to obtain the relative expression against hsa-miR-103a. The values obtained were multiplied by 1000 and then the results were plotted in logarithmic scale ${ }^{26}$ (Fig. 4i). All primers used were listed in the Supplementary Table 15.

Immunostaining. Cells were fixed in 4\% Paraformaldehyde (PFA, Sigma), permeabilized with $0.5 \%$ Triton X-100 (Sigma) in DPBS (ThermoFisher) and blocked with 5\% goat serum (ThermoFisher). All antibodies used in this study were described in Supplementary Table 16. For example, primary antibodies used: rabbit anti-KLF17 polyclonal (1:500, Sigma), mouse anti-TRA-1-60 IgM (1:300, BD). Primary antibody incubation was conducted overnight at $4{ }^{\circ} \mathrm{C}$ on shakers followed by incubation with secondary antibodies (1:400) for 1 hour. Secondary antibodies used in this study were goat anti-mouse IgM AF488 (1:400, ThermoFisher) or goat anti-mouse IgM AF647 (1:400, Invitrogen) for TRA-1-60, goat antirabbit IgG AF555 (1:400, ThermoFisher) or goat anti-rabbit IgG AF647 (1:400, ThermoFisher) for KLF17 (Supplementary Table 16). After labeling, cells were stained with 4',6-Diamidino-2-Phenylindole, Dihydrochloride (DAPI, 1:1000, ThermoFisher) for $30 \mathrm{~min}$. Images were taken by IX71 inverted fluorescent microscope (Olympus). For whole well (24well plates) scanning of TRA-1-60 positive colonies for primed condition, KLF17 positive colonies for naive condition, and KRT7 positive colonies for Extended Data Fig. 8k, DMi8 microscope (Leica) was used, and the number of colonies in each well was quantified using ImageJ. For Extended Data Fig. 9g, NR2F2 was used as a trophoblast marker as suggested by a recent study ${ }^{40}$.

Single-nucleus RNA-sequencing (snRNA-seq) of human reprogramming intermediates. For snRNA-seq experiments, day 0, day 4, day 8, day 12 primed, day 12 naive, day 16 
737 primed, day 16 naive, day 20 primed, day 20 naive, day 24 primed, day 24 naive, hiPSC 738 naive (passage 3), hiPSC primed (passage 20) and hiPSC naive (passage 20) were collected 739 and cryopreserved. These collected samples were then subjected to FACS, for D0, D4, D8, 740 D12 primed, D12 naive, D16 primed, D16 naive, D20 primed, D20 naive, D24 primed and 741 D24 naive samples were sorted for PI negative, TRA-1-85 positive cells to remove dead cells 742 and iMEF cells, while hiPSC primed (passage 3) and hiPSC naive (passage 3 and passage 20) 743 samples were sorted for PI negative, TRA-1-85 positive, CD13 negative, F11R positive, 744 TRA-1-60 positive, EPCAM positive cells to get rid of dead cells and iMEF cells as well as 745 differentiated cells. snRNA-seq library preparation was then prepared separately on each 746 timepoint, generating 14 libraries (Fig. 1a). Nuclei were prepared using the 'Frankenstein' 747 protocol for nuclei isolation from fresh and frozen tissue followed by 10x Genomics that can 748 be found in protocols.io. Briefly, cells were thaw and pelleted at 500xg for 5 minutes at $4{ }^{\circ} \mathrm{C}$. $749500 \mu \mathrm{L}$ of chilled Nuclei EZ Lysis Buffer supplemented with $0.2 \mathrm{U} / \mu 1$ RNase Inhibitor was 750 added to the pellet of cells and resuspended gently with a $1000 \mu \mathrm{L}$ bore tip and rest on ice for 7515 ' to complete lysis. The homogenate was filtered once using a $70 \mu \mathrm{m}$ Flowmi filter and 752 centrifuged at $500 \mathrm{xg}$ for 5 minutes at $4^{\circ} \mathrm{C}$. After removing the supernatant (leaving $50 \mu \mathrm{L}$ 753 behind) the nuclei pellet was washed with $1000 \mu \mathrm{L}$ of chilled Nuclei Wash and Resuspension 754 Buffer (1x PBS, 1.0\% BSA, $0.2 \mathrm{U} / \mu 1$ RNase Inhibitor). The nuclei were again pelleted at 755 756 $500 \mathrm{~g}$ for 5 minutes at $4^{\circ} \mathrm{C}$, remove supernatant leaving behind $\sim 50 \mu \mathrm{L}$ and gently resuspend nuclei in $1000 \mu \mathrm{L}$ Nuclei Wash and Resuspension Buffer. Nuclei were pelleted, supernatant removed and resuspended in $300 \mu \mathrm{L}$ of Nuclei Wash and Resuspension Buffer supplemented with DAPI $(10 \mu \mathrm{g} / \mathrm{mL})$. Nuclei suspension was filtered using a $40 \mu \mathrm{m}$ Flowmi filter, nuclei 759 integrity was visually inspected under a microscope, and proceeded with cytometric analysis 760 and sorting based on DNA content using $70 \mu \mathrm{m}$ nozzle, gating for single nucleus and sorting 761 directly into Reverse Transcription Buffer without RT Enzyme: $20 \mu \mathrm{L}$ RT Buffer, $3.1 \mu \mathrm{L}$ 762 TSO primer, $2 \mu \mathrm{L}$ Additive $\mathrm{B}$ and $30 \mu \mathrm{L} \mathrm{H}_{2} \mathrm{O}$. After sorting nuclei (1000-7000 nuclei 763 depending on sample), complete volume to $80 \mu \mathrm{L}$ with $\mathrm{H}_{2} \mathrm{O}$, add $8.3 \mathrm{uL}$ RT Enzyme C, 764 mixed and proceeded with chip loading. All the steps from forward were carried out as 765 described in the Chromium Single Cell 3' Reagent Kits User Guide (v3 Chemistry). 766 Sequencing was done on a Illumina NovaSeq 6000 using a paired-end 2x150 sequencing 767 strategy and aiming for 30,000 read-pairs per nucleus. Chromium barcodes were used for 768 demultiplexing and FASTQ files were generated from the mkfastq pipeline using the 769 Cellranger program (v3.0.2). Alignment to hg19 genome (GRCh37, CellRanger reference 770 version 1.2.0, genome build GRCh37.p13, which contained the Sendai virus KLF4, MYC and 
$771 \mathrm{SeV}$ sequences as extra chromosomes) and UMI counting were then performed using 772 Cellranger against Ensembl's GRCh37 genome annotation (version 82, including protein773 coding, lincRNA and antisense byotipes) containing the Sendai virus sequences as extra 774 transcripts. The endogenous expression of Yamanaka factors was quantified by only counting 775 sequencing reads against the 5' and 3' UTR regions of the endogenous OKSM transcripts.

776 Single cell RNA-sequencing (scRNA-seq) of human reprogramming intermediates. For 777 scRNA-seq experiments, day 0, day 3, day 7, day 13 primed, day 13 naive, day 21 primed, 778 day 21 naive, hiPSC primed (passage 3) and hiPSC naive (passage 3) were collected and 779 cryopreserved. These collected samples were then subjected to FACS, for D0, D3, D7, D13 780 primed, D13 naive, D21 primed and D21 naive samples were sorted for PI negative, TRA-178185 positive cells to remove dead cells and iMEF cells, while hiPSC primed (passage 3 ) and 782 hiPSC naive (passage 3) samples were sorted for PI negative, TRA-1-85 positive, CD13 783 negative, F11R positive, TRA-1-60 positive, EPCAM positive cells to get rid of dead cells 784 and iMEF cells as well as differentiated cells. Three samples were prepared in Extended Data 785 Fig. 1c) for subsequent library preparation, sample one contained cells isolated from D0, 3 786 and 7, samples two and three contained cells for primed (D13, D21, hiPSCs) and naive 787 reprogramming (D13, D21, hiPSCs) respectively, and a small number of mixed D0, 3 and 7 788 cells were added to sample two and three to capture the full reprogramming trajectories and 789 also to account for potential batch effects. The collected cells were isolated, encapsulated and 790 library constructed using Chromium controller (10x Genomics) as per the manufacturer's 791 instructions "Chromium Single Cell 3' Reagent Kit V2 User Guide", 10X Genomics 792 document number CG00052 Revision 3. A total of 12 cDNA amplification cycles were used. 793 A total of 16 cycles of library amplification were used. Sequencing was carried out using an 794 Illumina NextSeq 500 using SBS V2 chemistry in a high-output mode according to the 795 recommendations outlined by 10x Genomics "Chromium Single Cell 3' Reagent Kit V2 User 796 Guide", 10x Genomics document number CG00052 Revision 3, with the exception that the 797 second read was extended to $115 \mathrm{~b}$ instead of $98 \mathrm{~b}$. Libraries were diluted according to the 798 manufacturer's instruction "NextSeq 500 System User Guide" Illumina document number 79915046563 v02 and loaded at 1.8pM. Chromium barcodes were used for demultiplexing and 800 FASTQ files were generated from the mkfastq pipeline using the Cellranger program 801 (v2.1.0). Alignment and UMI counting were performed to the hg19 genome as per the 802 snRNA-seq. The same experimental procedure and the computational pipeline were also 
803

804

805

806

807

808

809

810

811

812

813

814

815

816

817

818

819

820

821

822

823

824

825

826

827

828

829

830

831

832

833

834

applied to generate the RSeT reprogramming scRNA-seq library shown in Extended Data Fig. 4a,b.

\section{scRNA-seq of day 21 fibroblast, naive and $\operatorname{iTSC}^{\mathrm{d} 8}$ reprogramming intermediates. For} Extended Data Fig. 9a, day 21 fibroblast, naive and $\mathrm{iTSC}^{\mathrm{d} 8}$ reprogramming intermediates were harvested and sorted for PI negative, TRA-1-85 positive cells to remove dead cells and iMEF cells. The collected cells were isolated, encapsulated and constructed using Chromium controller (10x Genomics) as per the manufacturer's instructions "Chromium Next GEM Single Cell 3' Reagent Kit V3.3 User Guide". Sequencing was done on an Illumina NovaSeq 6000 using a paired-end (R1 28bp and R2 87bp) sequencing strategy and aiming for 20,000 read-pairs per cell. Chromium barcodes were used for demultiplexing and FASTQ files were generated from the mkfastq pipeline using the Cellranger program (v3.1.0). Alignment and UMI counting were performed to the hg19 genome as per the scRNA-seq experiments.

snRNA-seq and scRNA-seq cell calling, quality control. To identify the cell-containing droplets, cell calling was performed on the raw_gene_bc_matrices generated by the Cellranger program as follows. All the cell barcodes are ranked in order of decreasing the number of total UMI counts. The $\log 10$-transformed total UMI counts (Y-axis) were then plotted against the log10-transformed rank (X-axis). The first "knee" point in this UMIbarcode rank plot represents a drastic drop in the total UMI counts, shifting from cellcontaining barcodes to the majority of non-cell-containing barcodes. To determine this "knee" point, a linear model was fitted on the UMI-barcode rank plot between the top $n_{\text {upper }}$ and $n_{\text {lower }}$ ranks. Barcodes that deviate negatively from the linear model by more than $k_{\text {cut }}$ on the Y-axis are then deemed to have passed the "knee" point and discarded. This cell calling procedure was performed on each library separately using $n_{\text {upper }}=100, n_{\text {lower }}=400, k_{\text {cut }}=$ 0.15 for the snRNA-seq and $n_{\text {upper }}=100, n_{\text {lower }}=500, k_{\text {cut }}=0.2$ for the scRNA-seq. This resulted in a total of 38,100 cells and 7,674 cells for the snRNA-seq and scRNA-seq respectively. Quality control was first performed at the cell level. Cells with (i) extremely high total UMI counts [nUMI], (ii) low number of expressed genes [nGene], (iii) high percentage mitochondrial genes [pctMT] or (iv) low percentage housekeeping genes, gene list from Tirosh et $\mathrm{al}^{41}$, [pctHK] were discarded. Cutoffs of $n U M I>15,000$, nGene $<1,200$ and $n U M I>50,000, n G e n e<1,800, p c t M T>12, p c t H K<10$ were applied to discard cells for the snRNA-seq and scRNA-seq respectively. No pctMT and pctHK cutoffs were applied in the case of snRNA-seq as there are very little mitochondrial or housekeeping genes detected. 
835 Next, quality control was performed at the gene level. Genes with (i) low log10 (average 836 UMI) [log10aveUMI] or (ii) do not have at least minUMI UMIs in at least minCell cells were 837 discarded. Cutoffs of log10aveUMI $<-2.5$, minUMI $=2$, minCell $=10$ and log10aveUMI $<-$ $8382, \min U M I=2, \operatorname{minCell}=10$ were applied to discard genes for the snRNA-seq and scRNA839 seq respectively. After quality control, 36,597 cells / 17,004 genes and 7,194 cells / 12,246 840 genes remain for the snRNA-seq and scRNA-seq respectively.

841 snRNA-seq ambient RNA removal. From the UMI-barcode rank plot in the snRNA-seq 842 libraries, we observed non-cell-containing barcodes with high total UMI counts (in the range 843 of 500-750 UMIs as compared to 20-50 UMIs in the scRNA-seq libraries), indicating 844 substantial ambient RNA contamination. To circumvent this, ambient RNA removal was then 845 performed using the decontx algorithm $^{42}$ in the celda package (v1.1.6). The decontx 846 algorithm assumes that there are $K$ cell populations and uses Bayesian variational inference to 847 infer the ambient RNA contamination as a weighted combination of the $K$ cell population 848 distributions. Thus, the algorithm requires the raw UMI counts and population membership 849 for each cell as input. To determine the cell population membership, we applied the Seurat 850 (v3.1.1) clustering pipeline ${ }^{20}$ using the following functions with default settings unless 851 otherwise stated: NormalizeData, FindVariableFeatures (with 2,000 features), ScaleData, 852 RunPCA. The cell clusters were then obtained using the FindNeighbors (using top 10 PCs) 853 and FindClusters (resolution $=0.5$ ) functions. The Seurat clustering pipeline was applied to 854 each snRNA-seq library separately and decontx was then performed on each library using the 855 default settings. A random seed of 42 was used throughout the entire analysis. snRNA-seq and scRNA-seq preprocessing and integration. To integrate both the snRNA857 seq and scRNA-seq datasets, we employed the Seurat v3 integration technique (v3.1.1) ${ }^{43}$. 858 Seurat v3 identifies "anchors" or pairwise correspondences between cells in the two datasets, 859 which is then used to harmonize the datasets. As part of the preprocessing step, the functions 860 NormalizeData (with default settings), FindVariableFeatures (using 1,500 features) were 861 applied to the snRNA-seq and scRNA-seq datasets separately. Furthermore, each cell was 862 assigned cell-cycle scores (S score and G2M score) and a cell-cycle phase using Seurat's 863 CellCycleScoring function. The FindIntegrationAnchors function (using 1,500 features) was 864 then executed to identify the anchors, followed by running the function IntegrateData on the 865 genes that are detected in both datasets. This resulted in an integrated single-cell dataset 
866

comprising 43,791 cells and 11,549 genes (Supplementary Table 1). The list of feature genes is in Supplementary Table 2.

scRNA-seq and snRNA-seq dimension reduction and trajectory inference. To represent the single-cell data in a concise manner, we applied several dimension reduction techniques using the anchor feature genes identified in the data integration step. Principal component analysis (PCA) was performed on the scaled gene expression using the RunPCA function in Seurat package (v3.1.1). Following that, Uniform Manifold Approximation and Projection (UMAP) and t-Distributed Stochastic Neighbor Embedding (t-SNE) were implemented on the top 14 PCs (determined using an elbow plot) via the RunUMAP and RunTSNE functions respectively. Diffusion maps were generated using the scanpy.pp.neighbors function (using the top 14 PCs generated above) and scanpy.tl.diffmap function in the scanpy package $(\mathrm{v} 1.4 .4 . p o s t 1)^{44}$. Force-directed layout was generated using the scanpy.tl.draw_graph function in the scanpy package using the ForceAtlas 2 layout and initialized using the UMAP coordinates. To infer the trajectories present in our single-cell data, we applied three different approaches. First, we applied the Cellular Trajectory Reconstruction Analysis using gene Counts and Expression (CytoTRACE, v0.1.0) algorithm ${ }^{18}$, which orders the single cells based on their differentiation potential. As our dataset comprises two different assays, we ran CytoTRACE in the integrated mode, which integrates the scRNA-seq and snRNA-seq data using the Scanorama method prior to calculating the differentiation potential. The raw counts were supplied as input and default settings were used. Second, we employed Monocle3 (v0.1.3) ${ }^{19}$ which learns a trajectory graph from a dimension reduction. In particular, we did a modification where we supplied the FDL dimension reduction calculated previously into Monocle 3 and ran the cluster_cells (using k $=30$ neighbours) and learn_graph functions in the monocle3 package to obtain an FDL-based monocle3 trajectory. Third, we used partitionbased graph abstraction (PAGA) ${ }^{21}$ which quantifies the connectivity between clusters of cells and generates an abstracted graph representing the trajectories observed during reprogramming. The PAGA algorithm was performed using the scanpy.tl.paga function in the scanpy package (v1.4.4.post1) using the Seurat cell clusters as input. The generation of the cell clusters will be described in the next section.

scRNA-seq and snRNA-seq cell clustering. The single cells were clustered using the FindNeighbors (using the top 14 PCs for consistency with the dimension reductions) and FindClusters function (resolution $=0.5$ ) in the Seurat (v3.1.1) package, which implements an 
898

899

900

901

902

903

904

905

906

907

908

909

910

911

912

913

914

915

916

917

918

919

920

921

922

923

924

925

926

927

928

929

unsupervised graph-based algorithm. This resulted in 21 clusters which are then labeled using a combination of letters and a number (e.g. cluster fm1) which were determined from the cell composition of the cluster (fm: fibroblast medium, mix: shared clusters, pr: primed reprogramming, nr: naive reprogramming, nic: novel intermediates cluster, re: refractory cells) and the ordering of the cell population along reprogramming trajectory.

snRNA-seq differential expression, identification of gene signatures. As the data integration introduces dependencies between data points, we chose to perform the differential expression analysis solely on the snRNA-seq. The snRNA-seq was chosen over the scRNAseq as the former has more cells and a larger number of detected genes. Prior to differential expression, we performed clustering on only the snRNA-seq using the procedure described earlier (using the top 12 PCs instead), generating 21 snRNA-clusters (Extended Data Fig. 2d). Pairwise differential expression between the 21 snRNA-clusters was performed using the Wilcoxon rank-sum test on the log-transformed gene expression. The Wilcoxon rank-sum test p-values are then adjusted for multiple testing using the Benjamini-Hochberg procedure to yield the false discovery rate (FDR). Genes are deemed differentially expressed if the $\log 2$ fold change (LFC) is $>1.5$ and the FDR is $<0.01$.

To identify gene signatures, we first define cluster-specific marker genes for each of the 21 snRNA-clusters. For each snRNA-cluster, we define marker genes as genes that have an average LFC (averaged across all 20 pairwise differential expressions) of $>1.5$ and we also require the genes to be differentially expressed in at least 14 of the 20 pairwise differential expressions. Hierarchical clustering was then performed on the Jaccard similarity of the marker genes (Extended Data Fig. 2f) to identify overlapping gene sets i.e. the gene signatures. Overall, we identified eight gene signatures (Supplementary Table 3), namely fibroblast (snRNA-fm1, snRNA-fm2, snRNA-fm3, snRNA-fm4); mixed (snRNA-mix); early-primed (snRNA-pr1); primed: snRNA-pr2, snRNA-pr3, snRNA-pr4); novel intermediates signature (snRNA-nic); naive (snRNA-nr1, snRNA-nr2, snRNA-nr3, snRNAnr4); nonReprog1 (snRNA-re1, snRNA-re3, snRNA-re4, snRNA-re5); nonReprog2 (snRNAre6). The marker genes for clusters snRNA-re2 and snRNA-fm5 were not used as there are very few genes. Furthermore, in the fibroblast, primed, naive and nonReprog1 gene signatures, which comprises marker genes from more than one cluster, we only pick genes that are called marker genes at least twice to be included in the gene signature. One mitochondrial gene was then removed, resulting in a total 504 genes across all eight gene 
930

931

932

933

934

935

936

937

938

939

940

941

942

943

944

945

946

947

948

949

950

951

952

953

954

955

956

957

958

959

960

signatures (Supplementary Table 3). We then determine the strength of each gene signature in every single cell by calculating the average expression of the genes of interest subtracted by the aggregated expression of a set of control genes ${ }^{41}$. The control genes were determined by binning all detected genes into 25 gene expression bins and 100 genes are then randomly selected from the same bin for each gene in the gene signature. Every single cell is then assigned to one of the 8 gene signatures based on the highest gene signature strength. This is then used to track the cell identity changes during reprogramming (Extended Data Fig. 2i). The same gene signature calculations were also applied to determine the strength of TE, EPI and PE gene signatures in each single cell (Fig. 3a and Extended Data Fig. 7g). Furthermore, gene signatures related to the $\mathrm{S}$ and G2M cell cycle phases were calculated to predict the cell cycle phase (Extended Data Fig. 1h). Single cells are assigned to the G1 phase if both $\mathrm{S}$ and G2M scores are less than zero. Otherwise, they are assigned either the S or G2M phase based on the higher of the S and G2M scores.

scRNA-seq analysis of RSeT reprogramming. The RSeT reprogramming (RR) scRNA-seq dataset was analyzed together with the FM, PR, and NR scRNA-seq counterparts (Supplementary Table 6). The raw UMI counts of all four scRNA-seq libraries were combined and subjected to the same quality control cutoffs: $n_{\text {upper }}=100, n_{\text {lower }}=500, k_{\text {cut }}=$ 0.2 for cell calling, $n U M I>50,000, n G e n e<1,800$, pctMT $>12$, pctHK $<10$ for cell QC and log10aveUMI $<-2, \operatorname{minUMI}=2, \operatorname{minCell}=10$ for gene QC. This resulted in 9,852 cells / 12,590 genes after quality control. Subsequently, the combined scRNA-seq datasets are analyzed using a similar workflow as the previous scRNA-seq and snRNA-seq dataset. The dataset was preprocessed using Seurat v3's NormalizeData (with default settings), FindVariableFeatures (using 1,500 features) functions. Next, PCA was performed, followed by other dimension algorithms (UMAP, t-SNE, diffusion maps and force-directed layout) using the top 15 PCs. We found that the RSeT cells follow the naive trajectory, but we also observed a primed-like cluster of cells, expressing primed-associated markers such as ZIC2 and NLGN4X (Extended Data Fig. 4a,b). We have previously shown that primed cells have a growth advantage over the naive population ${ }^{4}$ and hence this could be the reason that they become the dominant population in the RSeT medium over time. These results suggest that $\mathrm{RSeT}$ is a more permissive condition that allows the derivation of a continuum of pluripotent states $^{4,6}$. 
961

962

963

964

965

966

967

968

969

970

971

972

973

974

975

976

977

978

979

980

981

982

983

984

985

986

987

988

989

990

991

992

scRNA-seq analysis of day 21 reprogramming intermediates. The day 21 reprogramming intermediates scRNA-seq libraries are analyzed using a similar workflow as the previous scRNA-seq and snRNA-seq dataset (Supplementary Table 13). Briefly, quality control (QC) was performed at both cell and gene level with the following cutoffs: $n_{\text {upper }}=100, n_{\text {lower }}=$ $500, k_{\text {cut }}=0.2$ for cell calling, $n U M I>50,000$, nGene $<1,800$, pctMT $>12$, pctHK $<10$ for cell QC and log10aveUMI $<-2$, minUMI $=2$, minCell $=10$ for gene QC. This resulted in 10,518 cells / 12,611 genes after quality control. Subsequently, the dataset was preprocessed using Seurat v3's NormalizeData (with default settings), FindVariableFeatures (using 1500 features) functions. Next, PCA was performed, followed by other dimension algorithms (UMAP, t-SNE, diffusion maps and force-directed layout) using the top 15 PCs. We also applied cell clustering (using the same top $15 \mathrm{PCs}$ and resolution $=0.5$ ), identifying 13 clusters. These clusters are then labeled using a combination of letters and a number (e.g. cluster D21tr1) which were determined from the cell composition of the cluster (D21fm: fibroblast medium, D21nr: naive reprogramming, D21tr: TSC reprogramming) and the ordering of the cell population along reprogramming trajectory. The strength of the 8 gene signatures defined in this study is also calculated as per the previous scRNA-seq and snRNAseq dataset.

RNA-sequencing (RNA-seq) of reprogramming intermediates. For the bulk RNA-seq of the FACS-purified reprogramming intermediates (Extended Data Fig. 3), RNA extraction was performed using the RNeasy micro kit (Qiagen, Cat\#74004) from $\sim 2-20 \times 10^{4}$ cells with QIAcube (Qiagen). The concentrations of RNA were measured by a Qubit RNA HS Assay Kit (ThermoFisher, Cat\#Q32855) on a Qubit 2.0 Fluorometer (ThermoFisher). 25 ng of RNA was used for library construction with the SPIA kit (NuGen) and subsequently sequenced by HiSeq 1500 or HiSeq 3000 sequencer (Illumina). Sequencing libraries were single-end with $50 \mathrm{nt}$ length and a targeted number of reads of 20-30 million.

RNA-seq analysis of reprogramming intermediates. bulk RNA-sequencing reads generated in this study, O'Brien et $\mathrm{al}^{22}$. [D0 fibroblasts, $n=2(32 \mathrm{~F}$ and $55 \mathrm{~F}$ biological replicates)] and Liu et $\mathrm{al}^{4}$. [P3 t2iLGoY, P10 t2iLGoY, P3 RSeT, P10 RSeT, P3 NHSM, P10 NHSM, P3 5iLAF, P10 5iLAF; all conditions with $n=2(32 \mathrm{~F}$ and 55F)] were processed as follows: low-quality sequencing reads and were filtered and trimmed with Trimmomatic ${ }^{45}(\mathrm{v}$ 0.36, Phred score of 6 consecutive bases below 15, minimum read length of 36nt) and mapped to a custom version of hg19 human genome (with modifications described above in 
993 the scRNA-seq sequencing and processing section) with STAR (v 2.4.2a) ${ }^{46}$. Gene read 994 counting was performed with featureCounts (v1.5.2, unstranded) ${ }^{47}$ against the custom version 995 of Ensembl's GRCh37 annotation with modifications described above in the snRNA996 seq/scRNA-seq sequencing and processing section. From the resulting counts table, we 997 retained genes that have (i) at least 10 counts in one sample and (ii) at least 2 counts per 998 million (CPM) in at least two samples so as to remove the lowly expressed genes. Library 999 normalization was then performed using the rpkm function in the edgeR package (v3.24.3) 1000 with the arguments normalized.lib.sizes $=$ TRUE and prior.count $=1$ to yield fragments per 1001 kilobase per million (FPKM). Principal component analysis (PCA) was then performed on 1002 the $\log$-transformed $\log 2(\mathrm{FPKM}+1)$ on the top 500 most highly variable genes using the 1003 prcomp_irlba function in the irbla package (v2.3.3). To show the reprogramming trajectory 1004 in the 3D PCA plots, cubic splines were fitted independently on each PC using the splinefun 1005 function in base R (v3.5.1).

1006 Projection of bulk RNA-seq samples onto single-cell data. To project the bulk RNA-seq 1007 samples of FACS-purified reprogramming intermediates onto the single-cell data, we treat 1008 each bulk RNA-seq sample as a "single-cell" and performed the same Seurat v3 integration 1009 technique that was previously used to integrate both the snRNA-seq and scRNA-seq. The 1010 same procedure was applied with the exception that the arguments k.filter $=20$ and $\mathrm{k}$.score $=$ 101110 were supplied to the FindIntegrationAnchors function to adjust for the fact that the bulk 1012 RNA-seq contains a lot fewer samples (50 samples) than the single-cell counterpart. We then 1013 aggregate the gene expression of the combined gene expression as follows. For the bulk 1014 RNA-seq, samples were aggregated based on the media condition and timepoint. For the 1015 single cells, the scRNA-seq cells and non-reprogrammed cells were removed and the 1016 remaining single nucleus was aggregated based on the media condition and timepoint.

1017 Scoring of bulk RNA-seq samples using the primed/naive gene signatures and 1018 TE/EPI/PE signatures. For the bulk RNA-seq samples of reprogramming intermediates, we 1019 employ a simple scoring system to determine the strength of different gene signatures 1020 (Supplementary Table 5). To compute the scores for each sample, the gene expression of the 1021 gene set of interest was first divided by the maximum gene expression across all samples to 1022 obtain a scaled gene expression ranging from 0 to 1 . The scaled gene expression was then 1023 averaged across all the genes in the gene set to give the final score, which ranges from 0 to 1. 1024 This scoring system was applied to determine the strength of the primed and naive 
1025

1026

1027

1028

1029

1030

1031

1032

1033

1034

1035

1036

1037

1038

1039

1040

1041

1042

1043

1044

1045

1046

1047

1048

1049

1050

1051

1052

1053

1054

1055

1056

pluripotency using the genes in the primed and naive gene signatures determined from the single-cell data respectively. We also utilized gene sets that are highly expressed in the epiblast (EPI), primitive endoderm (PE) and trophectoderm (TE) based on the previous study $^{25}$. In particular, we obtained the top 100 genes, ordered by differential expression FDR in that study, for each of the three lineages across E5 to E7, giving rise to the ALL-EPI, ALLPE, and ALL-TE gene sets. Furthermore, we also extracted the top 100 genes for each embryonic day, giving rise to day-specific EPI (E5-EPI, E6-EPI, E7-EPI), PE and TE gene sets. These gene sets can be found in Supplementary Table 11. To validate this scoring approach, gene set enrichment analysis on each media/timepoint condition was performed as follows. Condition-specific differential expression was performed using the empirical Bayes quasi-likelihood F-tests in the edgeR package (v3.24.3) between the condition of interest and the average expression of the remaining conditions. Gene set enrichment analysis was then performed on the log fold changes from these differential expression results using the fgsea package (v1.8.0) with 10,000 permutations.

RNA-seq for characterization of iTSCs and iTSC-differentiated cells. For the bulk RNAseq of the iTSC and iTSC-differentiated cells, RNA-seq was performed with a multiplexing approach, using an 8 bp sample index ${ }^{48}$ and a $10 \mathrm{bp}$ unique molecular identifier (UMI) were added during initial poly(A) priming and pooled samples were amplified using a templateswitching oligonucleotide. The Illumina P5 (5' AAT GAT ACG GCG ACC ACC GA 3') and P7 (5' CAA GCA GAA GAC GGC ATA CGA GAT 3') sequences were added by PCR and Nextera transposase, respectively. The library was designed so that the forward read (R1) utilizes a custom primer (5' GCC TGT CCG CGG AAG CAG TGG TAT CAA CGC AGA GTA C $3^{\prime}$ ) to sequence directly into the index and then the $10 \mathrm{bp}$ UMI. The reverse read (R2) uses the standard R2 primer to sequence the cDNA in the sense direction for transcript identification. Sequencing was performed on the NextSeq550 (Illumina), using the V2 High output kit (Illumina, \#TG-160-2005) in accordance with the Illumina Protocol 15046563 v02, generating 2 reads per cluster composed of a 19 bp R1 and a 72 bp R2.

Analysis of the RNA-seq of iTSCs and iTSC-differentiated cells. The sequencing reads are demultiplexed using sabre (v1.0) using the barcodes-sample table, and allowing up to one mismatch per barcode, and a minimum UMI length of 9bp. The demultiplexed data has single reads per sample and UMIs are added to the read name. We use STAR (v2.5.2b) ${ }^{46}$ to align the reads to the GRCh37 Ensembl reference genome (v87). Read deduplication based on UMIs 
1057 was performed with je markdupes (v1.2) ${ }^{49}$ and transcript read counts calculated with 1058 featureCounts (v1.5.2) $)^{47}$. From the resulting counts table, lowly expressed genes were filtered 1059 and library normalization was performed as per the bulk RNA-seq analysis of reprogramming 1060 intermediates. We then compared the similarity of the transcriptomes of our iTSC, iTSC1061 derived EVT/STs with published transcriptomic datasets, namely (i) blastocyst-derived TSCs 1062 gene expression from Okae et $\mathrm{al}^{7}$. and Dong et $\mathrm{al}^{30}$; (ii) trophoblast organoids gene 1063 expression from Haider et $\mathrm{al}^{29}$. and Turco et $\mathrm{al}^{28}$. and (iii) single-cell gene expression (only 1064 Smart-seq2) of the fetal-maternal interface from Vento-Tormo et $\mathrm{al}^{27}$. The removeBatchEffect 1065 function in the limma package (v3.38.3) was applied to our dataset and each of the three sets 1066 of external datasets separately to account for technical differences, followed by Spearman 1067 correlation between the two datasets.

1068

Assay for transposase-accessible chromatin using sequencing (ATAC-seq). ATAC-seq 1069 samples were prepared as previously described ${ }^{50}$. Briefly, reprogramming intermediates and 1070 hiPSCs were isolated by FACS (Supplementary Table 4) and $\sim 65 \mathrm{k}$ cells were washed and 1071 lysed in ATAC-seq lysis buffer (10 mM NaCl, $3 \mathrm{mM} \mathrm{MgCl}_{2}, 0.1 \%$ IGEPAL CA-630, $10 \mathrm{mM}$

1072 Tris $\mathrm{pH}$ 7.4). The transposition reaction was then carried out by using $22.5 \mu$ l of UltraPure 1073 Distilled Water (ThermoFisher, Cat\#10977-015), $25 \mu 1$ of Tagment DNA Buffer (Illumina, 1074 Cat\#15027866) and $2.5 \mu \mathrm{l}$ of Tagment DNA Enzyme 1 (Illumina, Cat\#15027865) for each 1075 sample, and then incubated for $30 \mathrm{~min}$ at $37^{\circ} \mathrm{C}$, followed by immediate purification using a 1076 MinElute Reaction Cleanup Kit (Qiagen, Cat\#28204) according to the manufacturer's 1077 instructions. $11 \mu \mathrm{l}$ of transposed DNA, 25 $\mu \mathrm{l}$ of the NEBNext High-Fidelity 2x PCR Master 1078 Mix (Cat\#M0541S) and $1.25 \mu \mathrm{M}$ of the adaptor sequences as published previously ${ }^{50}$ were 1079 used in a $50 \mu \mathrm{l} \mathrm{PCR}$ reaction. PCR parameters were: $72^{\circ} \mathrm{C}$ for $5 \mathrm{~min}, 98^{\circ} \mathrm{C}$ for $30 \mathrm{~s}$ and 9 1080 cycles of $98^{\circ} \mathrm{C}$ for $10 \mathrm{~s}, 63^{\circ} \mathrm{C}$ for $30 \mathrm{~s}$ and $72^{\circ} \mathrm{C}$ for $1 \mathrm{~min}$. The prepared libraries were 1081 purified using a MinElute PCR purification kit (Qiagen, Cat\#28004) followed by Agencourt 1082 AMPure XP beads (Beckman Coulter, Cat\#A63880) according to the manufacturer's 1083 specifications, where library fragments ranging from 200 to $700 \mathrm{bp}$ were selected and 1084 sequenced on an Illumina HiSeq 1500 in 2x51 cycle paired-end mode.

1085 ATAC-seq preprocessing and alignment. ATAC sequencing reads (pair-end 51nt reads) 1086 were adaptor-trimmed and filtered by base quality and length using Cutadapt v $1.8^{51}$ using -a 1087 CTGTCTCTTATACACATCT, -A CTGTCTCTTATACACATCT, -q 20, and --minimum1088 length 18 options. Read pairs passing filters were mapped to the complete human genome 
1089 [hg19 human genome (UCSC version, December 2011)] using Bowtie2 with -X 2000, --no1090 mixed and --no-discordant options ${ }^{52}$. Mapped sample reads were filtered for multi-mappers 1091 (mapping quality < 10) and reads mapped to mitochondrial DNA using Jvarkit's ${ }^{53}$ samjs. 1092 PCR duplicates were discarded using Picard's (http://broadinstitute.github.io/picard) 1093 MarkDuplicates tool. Sequencing reads aligned to known genomic blacklisted regions were 1094 also not considered for further analysis ${ }^{54}$.

1095 ATAC-seq peak calling and exploratory analysis. Peak calling was performed on each 1096 biological replicate with MACS2 callpeak subcommand ${ }^{55}$ using --nomodel -f BAM --keep1097 dup all --gsize hs --shift -100 --extsize 200 --SPMR -B options. For downstream analysis we 1098 use an "intersect and rescue" approach. This approach consisted of intersecting each time 1099 point and reprogramming media biological replicates peak sets (bedtools intersect) ${ }^{56}$, 1100 subcommand (-wa -wb -F/-f 0.3) and then filtering those peaks with a fold change over 1101 background of more than 5 fold change (FC) and at least 3 FC in the other replicate. This 1102 created two intersection peaksets (major to 5 FC in replicate 1 and major to 3 FC in replicate 11032 and vice versa), which were then combined and merged with bedtools merge (a minimum 1104 of $1 \mathrm{bp}$ overlap). The union peakset of both replicates for each timepoint and reprogramming 1105 media was then reduced by merging all peaks within $100 \mathrm{bp}$. Finally, a consensus peak set of 1106 all time points and reprogramming media was created using bedtools merge as described 1107 above. Sequencing read counts for each biological replicate time point and media were 1108 produced using featureCounts ${ }^{47}(-\mathrm{p}-\mathrm{F}$ SAF), FPKMs calculated (peaks with less than 5 1109 FPKMs in at least 2 samples were discarded) then $\log _{2}$ transformed $\left(\log _{2}+1\right)$ and quantile 1110 normalised. Genome coverage plots were generated using wiggleplotr bioconductor 1111 package ${ }^{57}$. Principal component analysis (PCA) was then performed on the log2-transformed 1112 FPKM on all features using the prcomp_irlba function in the irbla package (v2.3.3). Human 1113 in vivo ICM and hESCs samples from $\mathrm{Wu}$ et $\mathrm{al}^{58}$, human blastocyst-derived TSCs (BT5) 1114 from Dong et $\mathrm{al}^{30}$ were processed as described above. We noted that RE of the fibroblast 1115 marker ANPEP became less accessible by day 7, accompanied by the downregulation of 1116 ANPEP gene expression. In contrast, This is followed by a gain of chromatin accessibility of 1117 regulatory elements and/or promoters RE of genes associated with shared pluripotency 1118 (PRDM14), primed pluripotency (SOX11) or naive pluripotency (DNMT3L) gain accessibility 1119 which coincides with the upregulation of these pluripotency genes (Extended Data Fig. 5d,e). 1120 We also observed naive-specific open chromatin regions in proximity or within the gene 1121 body of naive pluripotency factors such as KLF17, ZNF729, NANOG and POU5F1 (OCT4) 
1122 as were previously reported in ATAC-seq datasets of in vivo human embryos ${ }^{58,59}$ (Extended

1123 Data Fig. 5f). In particular, we found that the chromatin accessibility of two previously 1124 identified naive enhancers at the OCT4 and NANOG loci ${ }^{59}$, also detected in human inner cell 1125 mass $(\mathrm{ICM})^{58}$, became gradually accessible up to day 7 whilst the cells were still in FM. 1126 Following this, these regions lost accessibility in the primed intermediates and hiPSCs, while 1127 remaining open in naive cells (Extended Data Fig. 5f).

1128 Integration of bulk ATAC-seq samples with bulk RNA-seq samples. To integrate the bulk 1129 ATAC-seq profiles with the bulk RNA-seq samples, we first selected ATAC-seq peaks that 1130 are within an activity distance of $-100,10 \mathrm{bp}$ around the TSS of each gene and assigned these 1131 peaks to the corresponding gene. Next, we further integrate the two assays by performing 1132 upper quartile normalisation, which makes the transcript counts and peak intensities 1133 distributions comparable and the removeBatchEffect command in the limma package 1134 (v3.38.3) to the combined $\log _{2}$ transformed $\left(\log _{2}+1\right)$ ATAC/RNA dataset, specifying that 1135 the terminal timepoints, namely Fibroblast-D0, Primed, t2iLGoY, to be preserved using the 1136 design argument. PCA was then performed on this integrated dataset using the top 1000 most 1137 highly variable genes. To characterise gene expression of genes associated with identified 1138 cluster peaks (see details below); annotated peaks with no genes associated to (intergenic) 1139 were discarded and in cases of peaks assigned to the same gene, the peak closest to the gene's 1140 TSS was selected. Bulk RNA-seq gene read counts were processed as described above, $\log 2$ 1141 FPKMs $(\log 2+1)$ and z-scores across all conditions calculated. Gene ontology (GO) analysis 1142 of genes associated to each cluster was then performed using the Metascape ${ }^{60}$, web interface 1143 (https://metascape.org) on GO biological processes with default settings. The top 20 1144 enriched GO terms for each cluster are presented in Supplementary Table 8.

1145 ATAC-seq fuzzy cluster analysis. Processing of the read counts for fuzzy clustering and c1146 means clustering was performed as previously described ${ }^{11}$. In summary, sequencing read 1147 counts of each biological replicate were aggregated, FPKMs calculated discarding peaks with 1148 less than 10 in any condition then $\log _{2}$ transformed $\left(\log _{2}+1\right)$ and quantile normalised. Only 1149 peaks with a coefficient of variation across timepoints and media higher than $20 \%$ were 1150 considered for clustering. This peak subset was z-scaled and c-means fuzzy clustering ${ }^{23}$ was 1151 performed $(\mathrm{m}=1.243778,8$ clusters) (Supplementary Table 7). A cluster membership 1152 threshold of 0.8 was used for downstream analysis. 
1153 ATAC-seq peak annotation and Motif analysis. Cluster peaks were annotated using 1154 Homer's annotatePeaks subcommand ${ }^{61}$ and annotatr ${ }^{62}$. A motif enrichment analysis of cluster 1155 peaks was performed using Homer's findMotifsGenome (-size given) for known motifs 1156 (Supplementary Table 9).

\section{Statistics and reproducibility}

1158 For the sn/scRNA-seq experiments of the reprogramming roadmap, specific library 1159 information can be found in Fig. 1b and Supplementary Table 1. For time-resolved snRNA1160 seq experiments, a total of $\mathrm{n}=14$ biologically independent samples across 14 1161 media/timepoints were included. Each sample is then subjected to snRNA-seq. The 1162 media/timepoints (D: day, P: passage, fm: fibroblast medium, pr: primed reprogramming 1163 medium, $n r$ : naive reprogramming medium) are D0-fm $(n=1), D 4-f m(n=1), D 8-f m(n=1)$, 1164 D12-pr (n=1), D12-nr (n=1), D16-pr (n=1), D16-nr (n=1), D20-pr (n=1), D20-nr (n=1), D241165 pr (n=1), D24-nr (n=1), P3-nr (n=1), P20-pr (n=1), P20-nr (n=1). For the media-resolved 1166 scRNA-seq experiments, a total of $\mathrm{n}=9$ biologically independent samples across 9 1167 media/timepoints were included. The media/timepoints are D0-fm (n=1), D3-fm (n=1), D71168 fm (n=1), D13-pr (n=1), D13-nr (n=1), D21-pr (n=1), D21-nr (n=1), P10-pr (n=1), P10-nr $1169(\mathrm{n}=1)$. These samples are then pooled into three scRNA-seq libraries, which are the FM 1170 library (D0-fm, D3-fm, D7-fm samples), PR library (D0-fm, D3-fm, D7-fm, D13-pr, D21-pr, 1171 P10-pr samples), NR library (D0-fm, D3-fm, D7-fm, D13-nr, D21-nr, P10-nr samples). The 1172 total number of cells used in the final analysis was 43,791 (Fig. 1b-g, 3a and Extended Data 1173 Fig. 7g,h,8j). Detailed cell numbers for sn and scRNA-seq in each figure are as follows. Fig. $11741 \mathrm{~b}$ and Extended Data Fig. 1e-g,k-r: 43,791 cells across 17 libraries (3,713 D0-fm cells, 3,511 1175 D4-fm cells, 3,809 D8-fm cells, 2,472 D12-pr cells, 491 D12-nr cells, 4,506 D16-pr cells, 1176 2,578 D16-nr cells, 2,680 D20-pr cells, 1,858 D20-nr cells, 2,148 D24-pr cells, 1,121 D24-nr 1177 cells, 2,169 P3-nr cells, 3,009 P20-pr cells, 2,532 P20-nr cells, 2,402 FM cells, 2,506 PR 1178 cells, 2,286 NR cells); Fig. 1f and Extended Data Fig. 2a-c: 43,791 cells across 21 clusters 1179 (2,691 fm1 cells, 1,326 fm2 cells, $955 \mathrm{fm} 3$ cells, 1,098 fm4 cells, $862 \mathrm{fm} 5$ cells, 1,424 fm6 1180 cells, 1,474 mix cells, 1,756 pr1 cells, 3,069 pr2 cells, 646 pr3 cells, 1,042 nr1 cells, 879 nr2 1181 cells, 4,270 nr3 cells, 6,049 nr4 cells, 505 nic cells, 2,159 re1 cells, 2,005 re2 cells, 1,361 re3 1182 cells, 2,992 re4 cells, 7,138 re5 cells, 90 re6 cells); Fig. 1g: 43,791 cells across 8 gene 1183 signatures (8,714 fibroblast cells, 2,575 mixed cells, 2,365 early-primed cells, 3,970 primed 1184 cells, 610 novel-interm. cells, 10,563 naive cells, 14,820 nonReprog1 cells, 174 nonReprog2 
1185 cells); Extended Data Fig. 1h: 43,791 cells across 3 cell cycle phases (18,771 G1 cells, 1186 12,090 S cells, 12,930 G2M cells); Extended Data Fig. 2d: 43,791 cells across 21 snRNA1187 clusters (7,194 scRNA(unused) cells, 2,501 snRNA-fm1 cells, 1,197 snRNA-fm2 cells, 1,060 1188 snRNA-fm3 cells, 1,392 snRNA-fm4 cells, 984 snRNA-fm5 cells, 1,164 snRNA-mix cells, 1189 1,121 snRNA-pr1 cells, 638 snRNA-pr2 cells, 783 snRNA-pr3 cells, 1,592 snRNA-pr4 cells, 1190 1,143 snRNA-nr1 cells, 3,020 snRNA-nr2 cells, 4,498 snRNA-nr3 cells, 1,039 snRNA-nr4 1191 cells, 406 snRNA-nic cells, 2,416 snRNA-re1 cells, 1,160 snRNA-re2 cells, 1,156 snRNA1192 re3 cells, 6,530 snRNA-re4 cells, 2,690 snRNA-re5 cells, 107 snRNA-re6 cells); Extended 1193 Data Fig. 2e,h: For gene expression trends, the normalised gene expression was averaged 1194 across all cells within the same cluster prior to log transformation; Extended Data Fig. 2f-h: 1195 Pairwise DEGs between the 21 snRNA-clusters were determined using two-sided Wilcoxon 1196 rank-sum test with p-values adjusted for multiple testing using the Benjamini-Hochberg 1197 procedure, genes that (i) have an average LFC (averaged across all 20 pairwise differential 1198 expressions) of $>1.5$ and (ii) are differentially expressed (LFC $>1.5$ and FDR $<0.01$ ) in at 1199 least 14 of the 20 pairwise differential expressions are deemed cluster-specific marker genes 1200 for each of the 21 snRNA-clusters. Hierarchical clustering was then performed on the Jaccard 1201 similarity of these marker genes to identify eight gene signatures (504 genes in total, 52 1202 fibroblast genes, 67 mixed genes, 28 early-primed genes, 39 primed genes, 31 naive genes, 120354 novel-interm. genes, 58 nonReprog1 genes, 175 nonReprog2 genes). For scRNA-seq of 1204 RSeT reprogramming, specific library information can be found in Extended Data Fig. 4a and 1205 Supplementary Table 6. On top of the scRNA-seq experiments mentioned earlier, an 1206 additional $n=3$ biological independent samples across 3 timepoints were included, namely 1207 D13-rr (rr: RSeT reprogramming), D21-rr, P10-rr. These samples are then pooled into the RR 1208 library containing the D0-fm, D3-fm, D7-fm, D13-rr, D21-rr, P10-rr samples. The total 1209 number of cells used in the final analysis (which included cells from the FM, PR and NR 1210 libraries mentioned above) was 9,852 (Extended Data Fig. 4). Detailed cell numbers for 1211 scRNA-seq in each figure are as follows. Extended Data Fig. 4a: 9,852 cells across 4 libraries 1212 (2,402 FM cells, 2,506 PR cells, 2,286 NR cells, 2,658 RR cells). For scRNA-seq of day 21 1213 reprogramming intermediates, specific library information can be found in Fig. 4a and 1214 Supplementary Table 13. A total of $n=3$ biologically independent samples across 3 conditions 1215 were included. Each sample is then subjected to scRNA-seq. The conditions are D21 1216 fibroblast medium (D21fm, n=1), D21 naive reprogramming (D21nr, n=1), D21 TSC 1217 reprogramming (D21tr, $\mathrm{n}=1$ ). The total number of cells used in the final analysis was 10,518 1218 (Fig. 4a,b and Extended Data Fig. 9b-e). Detailed cell numbers for scRNA-seq of day 21 
1219

1220

1221

1222

1223

1224

1225

1226

1227

1228

1229

1230

1231

1232

1233

1234

1235

1236

1237

1238

1239

1240

1241

1242

1243

1244

1245

1246

1247

1248

1249

1250

1251

1252

reprogramming intermediates in each figure are as follows. Fig. 4a: 10,518 cells across 3 libraries (4,761 D21 fm cells, 2,801 D21nr cells, 2,956 D21tr cells); Extended Data Fig. 9c: 10,518 cells across 13 clusters $(89$ D21fm1 cells, 531 D21fm2 cells, 329 D21fm3 cells, 268 D21fm4 cells, 480 D21fm5 cells, 315 D21fm6 cells, 2,797 D21fm7 cells, 147 D21nr1 cells, 899 D21nr2 cells, 1,771 D21nr3 cells, 301 D21tr1 cells, 629 D21tr2 cells, 1,962 D21tr3 cells); Extended Fig 9b and Extended Data Fig. 9d: The marked D21tr1 containing 301 cells comprises 6 D21fm cells, 16 D21nr cells, 279 D21tr cells. For bulk RNA-seq of reprogramming intermediates, specific library information can be found in Extended Data Fig. 3f and Supplementary Table 5. $\mathrm{n}=2$ biological replicates were obtained for each condition except for day 13 primed $(n=3)$, day 13 naive $(n=3)$ and passage 3 primed $(n=4)$ (Fig. 2a and Extended Data Fig. 3b,f, 5b,c). For the scoring of primed and naive signatures, gene expression trends and Spearman correlation comparisons, the FPKM values were averaged across replicates prior to $\log 2+1$ transformation (Fig. $2 \mathrm{~b}$ and Extended Data Fig. $3 \mathrm{~g}, 6 \mathrm{f}, 7 \mathrm{~d}-\mathrm{f})$. Gene expression trends of genes associated with ATAC-seq peaks are shown as z-standardised values (Extended Data Fig. 6b,c). In Extended Data Fig. 7e, gene set enrichment analysis was then performed on the log fold changes from condition-specific differential expression results with 10,000 permutations. The p-values from the gene set enrichment were then corrected for multiple testing via the Benjamini-Hochberg procedure to yield the FDR. The product of the normalised enrichment score (NES) and -log10(FDR) [NES * $-\log 10(\mathrm{FDR})]$ is then plotted in the heatmap in Extended Data Fig. 7e. For bulk RNA-seq of iTSC-related samples, specific library information can be found in Supplementary Table 14. $\mathrm{n}=2$ biological replicates were obtained for each condition except for $\operatorname{iTSC}^{\mathrm{d} 21 \mathrm{n}}(\mathrm{n}=3)$, iTSC ${ }^{\mathrm{d} 8}$-EVT $(\mathrm{n}=4)$ and $\operatorname{iTSC}^{\mathrm{d} 21 \mathrm{n}}$-EVT $(\mathrm{n}=4)$ (Extended Data Fig. 8b, $10 \mathrm{~b}, \mathrm{i}, \mathrm{j})$. For the Spearman correlation comparisons, the FPKM values were averaged across replicates prior to $\log 2$ transformation (Fig. 4h and Extended Data Fig. 10k,1). For ATAC-seq of reprogramming intermediates, specific library information can be found in Supplementary Table 5. $\mathrm{n}=2$ biological replicates were obtained for each condition. For PCA, each replicate peak counts FPKMs were calculated (peaks with less than 5 FPKMs in at least 2 samples were discarded), $\log 2$ transformed $(\log 2+1)$ and quantile normalised (Fig. 2c and Extended Data Fig. 5a). For fuzzy clustering, replicate counts were aggregated for each peak, FPKMs calculated (discarding peaks with less than 10 FPKM in any condition), log2 transformed $(\log 2+1)$ and quantile normalised. Peaks with a coefficient of variation $<20 \%$ were discarded. This peak subset was z-scaled and c-means fuzzy clustering was performed $(\mathrm{m}=$ 1.243778, 8 clusters) (Supplementary Table 7). A cluster membership threshold of 0.8 was 
1253

1254

1255

1256

1257

1258

1259

1260

1261

1262

1263

1264

1265

1266

1267

1268

1269

1270

1271

1272

1273

1274

1275

1276

1277

1278

1279

1280

1281

1282

1283

1284

1285

1286

used for downstream analysis. The number of peaks per cluster is as follows: C1, 12024; C2, 7779; C3, 5077; C 4, 3334; C5, 9117; C6, 10129; C7, 4885; C8, 7739 (Fig. 2d). Cluster specific peak trends are shown as the mean +/- SD for each reprogramming media (Fig. 2d). P-values of motif enrichment analysis of cluster specific peaks are calculated based on a cumulative binomial distribution to then calculate the probability of detecting them in target sequences by chance (Fig. 2e). Chromatin accessibility trends for peak associated genes are shown as z-scaled across reprogramming stages calculated as described above (Extended Data Fig. 6b,c). In Fig. 2f, for TFAP2C KD experiments, two reprogramming rounds were performed and for each round of reprogramming, $n=3$ independent experimental replicates were transduced, reprogrammed and quantified separately for both scrambled controls and shTFAP2C reprogramming into either primed or naive hiPSCs. Primed: $p$ value $=0.09$, Naive: $\mathrm{p}$ value $=0.001$. Data are represented as mean \pm s.e.m., the significance is determined statistically by two-tailed unpaired Student's t-test. For GATA2 KD experiments, two reprogramming rounds $(n=2)$ were performed for primed reprogramming. For round $1: n=6$ independent experimental replicates were transduced, reprogrammed and quantified separately for both scrambled controls and shGATA2 reprogramming into either primed or naive hiPSCs. For round 2: $n=4$ independent experimental replicates for scrambled control primed reprogramming, $\mathrm{n}=5$ independent experimental replicates for scrambled control naive reprogramming, $\mathrm{n}=5$ independent experimental replicates for shGATA2 primed reprogramming and $\mathrm{n}=5$ independent experimental replicates for shGATA2 naive reprogramming. Primed: $\mathrm{p}=2.33 \times 10^{-12}$, Naive: $\mathrm{p}=1.03 \times 10^{-5}$. Data are represented as mean \pm s.e.m., the significance is determined statistically by two-tailed unpaired Student's t-test. For Fig. $3 \mathrm{c}-\mathrm{e}$, these experiments were repeated $\mathrm{n}=4$ biological replicates ( 4 independent experiments from two donors) with similar results and representative images were shown in the figures. For Fig. 3f, n=3 biological replicates, 3 independent iTSC cell lines were injected into three mice, and similar results were obtained, and representative results were shown in the figure. For Fig. 3g, 4 lesions were generated from iTSC lines, harvested and analysed, similar results were obtained and representative images are shown ( $\mathrm{n}=4$ biological replicates) For Fig. $4 d-e$, the experiments were repeated independently ( $\mathrm{n}=4$ biological replicates) with similar results and representative results were shown in the figures. For Fig. 4f-g, the experiments were repeated with 4 iTSC cell lines obtained from the two donors were independently differentiated into STs and EVTs) with similar results and representative images were shown in the figures $(n=4$ biological replicates). For Fig. $4 i$, the experiments were repeated with 4 independent cell lines (obtained from the two donors) and each of the 4 
1287

1288

1289

1290

1291

1292

1293

1294

1295

1296

1297

1298

1299

1300

1301

1302

1303

1304

1305

1306

1307

1308

1309

1310

1311

1312

1313

1314

1315

1316

1317

1318

1319

1320

experiments were performed in 2 technical replicates with similar results and representative plots were shown in the figure ( $\mathrm{n}=4$ biological replicates $\mathrm{x} 2$ technical replicates). For Fig. $4 \mathrm{k}$, $\mathrm{n}=3$ independent cell lines were injected to three mice, and similar results were obtained and a representative image is shown. For Fig. 41, the serum of two independent experiments (2 iTSC lines injected, 1 line per mouse)) were measured in 2 technical replicates $(n=2$ biological replicates $\times 2$ technical replicates). Representative results were shown in the figure. For Fig. 4m, 4 lesions were generated, harvested and analysed, similar results were obtained and representative images are shown ( $\mathrm{n}=4$ biological replicates). For Extended Data Fig. 1a, more than 10 reprogramming experiments using two different donors were performed with similar results. Representative phase-contrast images are shown in the figure. For Extended Data Fig. 1b, representative images were shown from staining of $n=2$ biological replicates. For Extended Data Fig. 3d, 4 experiments were independently performed (from two donors) with similar results and representative images were shown in the figures $(n=4$ biological replicates). For Extended Data Fig. 3e, n=2 biological replicates (from two donors) were used for analysis in this figure. For Extended Data Fig. $6 \mathrm{~g}$, the relative expression of TFAP2C and GATA2 were measured in $\mathrm{n}=2$ independent experiments with technical replicates. Representative results were shown in the figure. For Extended Data Fig. 8a, the experiments were repeated independently with $n=2$ biological replicates (from two donors) with similar results and representative images were shown in the figures. For Extended Data Fig. 8c, these experiments were repeated $n=4$ biological replicates ( 4 independent experiments from two donors) with similar results and representative images were shown in the figures. For Extended Data Fig. 8d, fusion index was used to quantify the efficiency of cell fusion, which is calculated by using the number of nuclei counted in the syncytia minus the number of syncytia, then divided by the total number of nuclei counted. The quantification was performed on $\mathrm{n}=5$ cell clusters counted randomly and independently across ST cells differentiated from two iTSC lines (obtained from two different donors) with similar results and representative results were shown in the figure. $\mathrm{p}=1.60 \times 10^{-7}$, data are represented as mean \pm s.e.m., the significance is determined statistically by two-tailed unpaired Student's ttest. For Extended data Fig. 8e, the conditioned media from $n=6$ biological replicates ( 6 independent cell lines from 2 different donors were differentiated into STs) were tested for hCG pregnancy tests and similar results were obtained from such tests, and representative results were shown in the figure. For Extended Data Fig. 8f, the conditioned media of two independent experiments (from two donors) were measured in 2 technical replicates $(\mathrm{n}=2$ biological replicates x 2 technical replicates). Representative results were shown in the figure. 
1321 For Extended Data Fig. 8g, the serum of two independent experiments (2 iTSC lines injected, 13221 line per mouse)) were measured in 2 technical replicates $(n=2$ biological replicates $\times 2$ 1323 technical replicates). Representative results were shown in the figure. For Extended Data Fig. 1324 8h, 4 lesions were generated, harvested and analysed ( $\mathrm{n}=4$ biological replicates). For 1325 Extended Data Fig. 8i, 4 lesions were generated from iTSC lines, harvested and analysed, 1326 similar results were obtained and representative images are shown ( $\mathrm{n}=4$ biological replicates). 1327 For Extended Data Fig. 8k, n=3 independent experiments for unenriched and CD70 low cells 1328 were performed and $\mathrm{n}=2$ for CD70 high cells. For Extended Data Fig. 9g, the experiments were repeated independently with $n=2$ biological replicates (from two donors) with similar results and representative images were shown in the figures. For Extended Data Fig 9h, the relative expression of NANOG, ZIC2, KLF17, DPPA3, GATA2 and KRT7 were measured in $\mathrm{n}=3$ independent experiments with technical replicates. For Extended Data 10a, the experiments were repeated with $n=6$ biological replicates $(3$ independent cell lines derived from each of the two donors) with similar results and representative images were shown in the figure. For Extended Data Fig. 10c, fusion index was used to quantify the efficiency of cell fusion, which is calculated by using the number of nuclei counted in the syncytia minus the number of syncytia, then divided by the total number of nuclei counted. The quantification was performed on $\mathrm{n}=5$ cell clusters counted randomly and independently across ST cells differentiated from two iTSC lines (obtained from two different donors) with similar results and representative results were shown in the figure. $\mathrm{p}=3.95 \times 10^{-7}$, data are represented as mean \pm s.e.m., the significance is determined statistically by two-tailed unpaired Student's t-test. For Extended Data Fig. 10d, the conditioned media from $n=6$ biological replicates (6 independent cell lines from 2 different donors were differentiated into STs) were tested for hCG pregnancy tests and similar results were obtained from such tests, and representative results were shown in the figure. For Extended Data Fig. 10e, the conditioned media of two independent experiments (from two donors) were measured in 2 technical replicates $(\mathrm{n}=2$ biological replicates $\mathrm{x} 2$ technical replicates). Representative results were shown in the figure. For Extended Data Fig. 10f-h, the experiments were repeated independently with $\mathrm{n}=4$ biological replicates with similar results and representative images were shown in the figure. For Extended Data Fig. 10m, 4 lesions were generated from iTSC lines, harvested and analysed ( $n=4$ biological replicates). For Supplementary Table 7, n=2 biological replicates (from two donors) were used for data analysis presented in this supplementary table. GO Enrichment p-values are calculated based on an accumulative 1354 hypergeometric distribution, and adjusted for multiple testing (q-values) using Benjamini- 
1355 Hochberg adjustment. For Supplementary Table 8, $\mathrm{n}=2$ biological replicates (from two 1356 donors) were used in this supplementary table. Motif enrichment P-values are calculated 1357 based on a cumulative binomial distribution. As described in Heinz S., et al ${ }^{61}$, the statistics 1358 assess the occurrence of motifs in target sequences vs a random background. From these 1359 motif occurrences it then calculates the probability of detecting them in target sequences by 1360 chance. The software used for these calculations is described in the Methods section.

1361 Reporting Summary. Further information on research design is available in the Nature 1362 Research Reporting Summary linked to this paper.

\section{Data availability}

1364 We developed an interactive online tool (http://hrpi.ddnetbio.com/) to facilitate easy exploration of the dataset and download of all processed datasets. Raw and processed next generation sequencing datasets were deposited at the NCBI Gene Expression Omnibus

1367 (GEO) repository under accession numbers: GSE150311: scRNA-seq experiments of 1368 intermediates during human primed and naive reprogramming; GSE150637: scRNA-seq experiments of day 21 reprogramming intermediates cultured under fibroblast condition, naive pluripotent and trophoblast stem cell conditions; GSE147564: snRNA-seq experiments of intermediates during human primed and naive reprogramming; GSE147641: ATAC-seq experiments of intermediates during human primed and naive reprogramming; GSE150590: ATAC-seq experiments of induced trophoblast stem cells; GSE149694: bulk RNA-seq experiments of intermediates during human primed and naive reprogramming; GSE150616: bulk RNA-seq experiments of induced trophoblast stem cells and their derived placenta subtypes. Source Data for four Figures and ten Extended Data Figures are provided within the online content of this paper.

\section{Code availability}

1379 All data were analysed with standard programs and packages as detailed above. Scripts can 1380 be found at https://github.com/SGDDNB/hrpi.

1381 34. Liu, X., Nefzger, C. \& Polo, J. Establishment and maintenance of human 1382 naive pluripotent stem cells by primed to naive conversion and reprogramming of 1383 fibroblasts. Protocol Exchange (2017) doi:10.1038/protex.2017.099. 
35. Guo, G. et al. Naive Pluripotent Stem Cells Derived Directly from Isolated Cells of the Human Inner Cell Mass. Stem Cell Reports 6, 437-446 (2016).

36. Pastor, W. A. et al. Naive Human Pluripotent Cells Feature a Methylation (2016).

37. Larcombe, M. R. et al. Production of High-Titer Lentiviral Particles for Stable Genetic Modification of Mammalian Cells. Methods Mol. Biol. 1940, 47-61 (2019).

38. Qiu, P. et al. Extracting a cellular hierarchy from high-dimensional cytometry data with SPADE. Nat. Biotechnol. 29, 886-891 (2011).

39. Nefzger, C. M. et al. A Versatile Strategy for Isolating a Highly Enriched

Population of Intestinal Stem Cells. Stem Cell Reports 6, 321-329 (2016).

40. Meistermann, D. et al. Spatio-temporal analysis of human preimplantation development reveals dynamics of epiblast and trophectoderm. doi:10.1101/604751.

41. Tirosh, I. et al. Dissecting the multicellular ecosystem of metastatic melanoma by single-cell RNA-seq. Science 352, 189-196 (2016).

42. Yang, S., Corbett, S. E., Koga, Y., Wang, Z. \& Johnson, W. E.

Decontamination of ambient RNA in single-cell RNA-seq with DecontX. BioRxiv (2019).

43. Stuart, T. et al. Comprehensive Integration of Single-Cell Data. Cell 177, 1888-1902.e21 (2019).

44. Wolf, F. A., Angerer, P. \& Theis, F. J. SCANPY: large-scale single-cell gene expression data analysis. Genome Biol. 19, 15 (2018).

45. Bolger, A. M., Lohse, M. \& Usadel, B. Trimmomatic: a flexible trimmer for Illumina sequence data. Bioinformatics 30, 2114-2120 (2014).

46. Dobin, A. et al. STAR: ultrafast universal RNA-seq aligner. Bioinformatics 
29, 15-21 (2013).

47. Liao, Y., Smyth, G. K. \& Shi, W. featureCounts: an efficient general purpose program for assigning sequence reads to genomic features. Bioinformatics 30, 923-930 (2014). human microglial phenotypes in Alzheimer's disease are controlled by amyloid plaque phagocytosis through Hifla. bioRxiv (2019). versatile suite to handle multiplexed NGS libraries with unique molecular identifiers. BMC Bioinformatics 17, 419 (2016).

50. Buenrostro, J. D., Giresi, P. G., Zaba, L. C., Chang, H. Y. \& Greenleaf, W. J.

51. Martin, M. Cutadapt removes adapter sequences from high-throughput sequencing reads. EMBnet.journal 17, 10 (2011). Nat. Methods 9, 357-359 (2012). (2015).

54. ENCODE Project Consortium. An integrated encyclopedia of DNA elements in the human genome. Nature 489, 57-74 (2012). 55. Feng, J., Liu, T., Qin, B., Zhang, Y. \& Liu, X. S. Identifying ChIP-seq enrichment using MACS. Nat. Protoc. 7, 1728-1740 (2012).

56. Quinlan, A. R. \& Hall, I. M. BEDTools: a flexible suite of utilities for 
comparing genomic features. Bioinformatics 26, 841-842 (2010).

57. Alasoo, K. et al. Transcriptional profiling of macrophages derived from monocytes and iPS cells identifies a conserved response to LPS and novel alternative transcription. Sci. Rep. 5, 12524 (2015).

58. $\mathrm{Wu}$, J. et al. Chromatin analysis in human early development reveals epigenetic transition during ZGA. Nature 557, 256-260 (2018).

59. Pastor, W. A. et al. TFAP2C regulates transcription in human naive pluripotency by opening enhancers. Nat. Cell Biol. 20, 553-564 (2018).

60. Zhou, Y. et al. Metascape provides a biologist-oriented resource for the analysis of systems-level datasets. Nat. Commun. 10, 1523 (2019).

61. Heinz, S. et al. Simple combinations of lineage-determining transcription factors prime cis-regulatory elements required for macrophage and B cell identities. Mol. Cell 38, 576-589 (2010).

62. Cavalcante, R. G. \& Sartor, M. A. annotatr: genomic regions in context. Bioinformatics 33, 2381-2383 (2017).

\section{Extended Data Fig. Legends}

Extended Data Fig. 1 | Experimental designs, analysis pipelines for single-nucleus and single-cell RNA-sequencing. a, Morphological changes of cells undergoing reprogramming in FM: Fibroblasts Medium; PR: Primed Reprogramming; NR: Naive Reprogramming. (FM: D0, 3, 7), PR (D13, D21, hiPSCs) and NR (D13, D21, hiPSCs), $n>10$, Scale bar, 500 $\mu \mathrm{m}$. b, Immunostaining at early stages (FM: D0, 3, 7), during PR (D13, D21) and NR (D13, D21) with TRA-1-60 for primed colonies, KLF17 for naive colonies and DAPI for nuclei staining, $n=2$. Scale bar, $50 \mu \mathrm{m}$. c, Experimental design for single-cell RNA-seq (scRNA-seq) libraries. FM(scRNA-seq)/fm(snRNA-seq): Fibroblasts Medium; PR/pr: Primed Reprogramming; NR/nr: Naive Reprogramming; iMEF: irradiated Mouse Embryonic Fibroblasts. d, Singlenucleus (sn) and single-cell (sc) RNA-seq data analysis strategy (see Methods for details). e, 
1461 Representation of integrated snRNA-seq and scRNA-seq experiments (43,791 cells) on FDL.

1462 f-g, Primed and naive libraries on FDL. h, FDL showing cells in predicted stages of the cell 1463 cycle. i, Reprogramming trajectories on FDL highlighting cells within each timepoint. j, 1464 Expression of genes associated with primed pluripotency (NLGN4X) and naive pluripotency 1465 (DPPA5) on FDL. k-r, PCA (k-p), diffusion maps (q) and UMAP (r) of sn/scRNA-seq data.

1466 For more details on sample number and statistics, please see statistics and reproducibility 1467 section.

Extended Data Fig. 2 | Resolving the molecular hallmarks of primed and naive reprogramming trajectories. a, Unsupervised clustering projected onto the FDL shown in 1471 Fig. 1 (43,791 cells). fm1-fm6: fibroblast and early reprogramming intermediates cell 1472 clusters; mix: shared cell cluster; pr1-pr3: primed reprogramming cell clusters; nr1-nr4: naive 1473 reprogramming cell clusters; nic: novel intermediate cell cluster; re1-re6: refractory cell 1474 clusters. $\mathbf{b}$, snRNA-seq timepoint/library contribution (composition and cell number) towards 1475 each cell cluster. c, PAGA trajectory inference on diffusion maps. d, snRNA-seq clusters, 1476 used to define gene signatures, on FDL. e, Dotplot showing the expression of mesenchymal and epithelial (MET) associated genes across cell clusters. f, Jaccard similarity of snRNA-seq cluster-specific genes. Cluster-specific genes are then grouped to define the eight gene signatures, highlighted at the bottom. g, Defined gene signatures on FDL. h, Gene expression heatmap of the primed or naive pluripotency signatures across the cell clusters (coloured arrows indicate known marker genes). i, Area plots showing the transition and activation of the defined signatures during primed and naive reprogramming over time. For more details on sample number and statistics, please see statistics and reproducibility section.

Extended Data Fig. 3 | Isolation and characterisation of intermediates during reprogramming into several naive human induced pluripotent states. a, Identification of cell surface markers for the isolation of primed and naive reprogramming intermediates. $\mathbf{b}$, PCA of bulk RNA-seq data of isolated intermediates during primed and naive reprogramming, $n \geq 2$. c, Experimental designs for the generation, isolation, and profiling of intermediates during reprogramming into several naive human induced pluripotent states. d, Morphological changes during reprogramming under naive 5iLAF, NHSM, and ReST culture conditions (see Methods), $n=4$. Scale bar, $500 \mu \mathrm{m}$. e, Visualisation of flow cytometry profiles 1493 (SPADE tree) of intermediates during reprogramming, $n=2$. f, PCA of RNA-seq of primed 
1494

1495

1496

1497

1498

1499

1500

1501

1502

1503

1504

1505

1506

1507

1508

1509

1510

1511

1512

1513

1514

1515

1516

1517

1518

1519

1520

1521

1522

1523

1524

1525

1526

1527

and several types of naive reprogramming intermediates (see Methods), $n \geq 2$. g, Heatmap showing gene expression profiles of primed and naive pluripotency signatures genes (defined in sn/scRNA-seq analysis) across reprogramming intermediates and hiPSCs derived under all different culture conditions, $n \geq 2$. For more details on sample number and statistics, please see statistics and reproducibility section.

Extended Data Fig. 4 | Single-cell profiling of the reprogramming pathway into naive RSeT state. a, FDL of fibroblast, primed, naive t2iLGoY and RSeT scRNA-seq libraries, naive RSeT scRNA-seq libraries (9,852 cells, see Methods). b, Expression profile of genes associated with human fibroblasts (ANPEP), shared pluripotency (NANOG), primed pluripotency (ZIC2, NLGN4X) and naive pluripotency (DNMT3L, DPPA5) on FDL. For more details on sample number and statistics, please see statistics and reproducibility section.

\section{Extended Data Fig. 5 | Dynamics of chromatin state transitions during reprogramming} into primed and naive human induced pluripotency. a, PCA plot of ATAC-seq nucleosome-free signals, PC1 vs PC3 related to Fig. 2c. ATAC-seq was performed using isolated reprogramming intermediates and hiPSCs from FM (D0, D3, D7), PR (D13, D21, P3, P10), NR (D13, D21, P3, P10), n=2. FM: Fibroblasts Medium (Black); PR: Primed Reprogramming (Orange); NR: Naive Reprogramming (Blue). b-c, PCA plot of the integration of RNA-seq and ATAC-seq experiments $(n \geq 2)$. d-e, ATAC-seq and corresponding RNA-seq tracks of primed and naive reprogramming intermediates for Fibroblast marker, ANPEP; Shared pluripotency marker, PRDM14; Primed-specific pluripotency marker SOX11; Naive-specific pluripotency marker DNMT3L. Model of each gene is shown: coding sequences, light blue boxes, and exons, dark blue boxes; introns are shown as light blue connecting lines. f, Naive-reprogramming-specific ATAC-seq signals in light grey) around core pluripotency factors NANOG and POU5F1 (OCT4), naivereprograming-specific KLF17 and ZNF729 in primed and naive reprogramming intermediates and hiPSCs compared to human ICM and primed hESCs ATAC-seq data ${ }^{58}$. For more details on sample number, please see statistics and reproducibility section. For more details on sample number and statistics, please see statistics and reproducibility section.

Extended Data Fig. 6 | Features of accessible chromatin landscape during reprogramming into primed and naive human induced pluripotency. a, Proportion of genomic regions in each of the ATAC-seq clusters. b, Averaged chromatin accessibility (z- 
1528

1529

1530

1531

1532

1533

1534

1535

1536

1537

1538

1539

1540

1541

1542

1543

1544

1545

1546

1547

1548

1549

1550

1551

1552

1553

1554

1555

1556

1557

1558

1559

1560

1561

scaled, $n=2$ ) and gene expression (z-scaled, $n \geq 2$ ) of one representative gene from each of the ATAC-seq peak clusters. c, Standardized gene expression (averaged z-scaling) of genes associated with ATAC-seq cluster peaks (see Methods). d, TF motif enrichment analysis of the ATAC-seq peak clusters. Motif enrichment (- $\log P$ value) heatmap by colour and the size the percentage $(\%)$ of sequences in the cluster featuring the motif. Red arrow points to OCT4/SOX2/NANOG/KLF4 motifs in transient ATAC-seq cluster (C3), Blue arrow=and enrichment of TE-associated TFs TFAP2C/GATA2 (C7 and C8) are indicated by blue arrows. e, Gene expression heatmap TFs identified in the motif enrichment analysis in d. f, TFAP2C and GATA2 gene expression during primed and naive reprogramming. g, qRT-PCR analysis of shTFAP2C and shGATA2 compared to scrambled controls, $n=2$. For more details on sample number and statistics, please see statistics and reproducibility section.

\section{Extended Data Fig. 7 | Uncovering the transcriptional programs of human fibroblast} reprogramming into naive induced pluripotency. a-b, Primed and naive scores, using gene signatures defined in this study (Fig. 1g), on human preimplantation embryos at indicated embryonic stages based on scRNA-seq experiments from published studies ${ }^{24,25}$. c, EPI, PE and TE signatures score at indicated embryonic stages ${ }^{25}$. d, EPI, PE, TE gene signatures ${ }^{25}$ from embryonic (E) day 5, 6, 7 on intermediates and hiPSCs reprogrammed under primed and different naive culture conditions (see Methods). e, Gene set enrichment analysis (GSEA, see methods) of the EPI, PE and TE gene signatures in reprogramming intermediates and hiPSCs reprogrammed under primed and several naive culture conditions. f, EPI, PE and TE gene signatures scores in reprogramming intermediates and hiPSCs reprogrammed under primed and several naive culture conditions. We used a combined gene signature across E5 to E7 for each lineage (see Methods). g, EPI and PE signatures on FDL with single-cell trajectories constructed using Monocle3 (43,791 cells), related to Fig. 3a. h, Scoring of novelintermediate signatures defined in this study (Extended Data Fig. 2f,g) on human preimplantation embryos of different lineages at indicated embryonic stages based on scRNA-seq experiments from published studies ${ }^{24,25}$. For more details on sample number and statistics, please see statistics and reproducibility section.

Extended Data Fig. 8 | Characterisation of iTSC ${ }^{\text {d21n }}$. a, Immunostaining of fibroblast, primed, naive t2iLGoY hiPSCs with P63, TFAP2C, GATA2, KRT7, $n=2$. Scale bar, $100 \mu \mathrm{m}$. b, Gene expression of trophoblast genes in fibroblasts, primed, naive t2iLGoY hiPSCs, $\mathrm{iTSC}^{\mathrm{d} 21 \mathrm{n}}$ and TSCs derived from a human blastocyst $\left(\mathrm{TSC}^{\text {blast }}\right)^{7}$ and first-trimester placental 
1562 trophoblast $\left(\mathrm{TSC}^{\mathrm{CT}}\right)^{7}$, mean of replicates, $n=2$. c, Phase-contrast image of ST and EVT cells 1563 differentiated from $\operatorname{iTSC}^{\mathrm{d} 21 \mathrm{n}}, n=4$. Scale bar, $100 \mu \mathrm{m}$. d, Fusion index of iTSC ${ }^{\mathrm{d} 21 \mathrm{n}}-\mathrm{ST}$ and $1564 \mathrm{iTSC}^{\mathrm{d} 21 \mathrm{n}}, n=5$, data are represented as mean \pm s.e.m., $p$ values by two-tailed unpaired 1565 Student's $t$-test. e, Representative results for OTC hCG pregnancy test for media of ST cells 1566 differentiated from iTSC ${ }^{\mathrm{d} 21 \mathrm{n}}$ and control media, $n=6 . \mathbf{f}, \mathrm{hCG}$ levels in iTSC ${ }^{\mathrm{d} 21 \mathrm{n}}$ and iTSC ${ }^{\mathrm{d} 21 \mathrm{n}}$ 1567 ST conditioned media, detected by ELISA, $n=4$. g, hCG level in mouse blood serum detected 1568 by ELISA, $n=4$. h, Lesions harvested from subcutaneously engrafted $\mathrm{iTSC}^{\mathrm{d} 2 \mathrm{n}}$ in NOD-SCID 1569 mice, $n=4$. i, Hematoxylin and eosin, and immunohistochemical staining of KRT7 in the 1570 lesions from $\mathbf{h}$, no evident lesions were observed in vehicle controls, $n=4$. Scale bar, $200 \mu \mathrm{m}$. $1571 \mathrm{j}$, Distinct level of CD70 expression in naive and TE populations (indicated by blue arrows) 1572 on FDL projection of sn/scRNA-seq datasets. k, Quantification of KRT7+ colony clusters 1573 after 9 days of transitioning into TSC media of unenriched, CD70 high and CD70 low 1574 populations, $n=2-3$ independent experiments, data are represented as mean \pm s.e.m., $p$ values 1575 by two-tailed unpaired Student's $t$-test. Representative images of whole-well scans (top

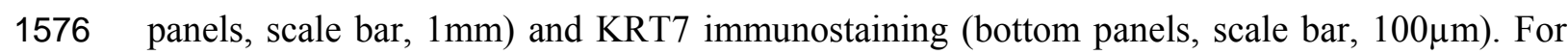
1577 more details on sample number and statistics, please see statistics and reproducibility section.

\section{Extended Data Fig. 9 | Cellular heterogeneity of fibroblast and iTSC ${ }^{\mathrm{d} 8}$ reprogramming} intermediates revealed by scRNA-seq. a, Experimental designs and preparation of singlecell RNA-seq (scRNA-seq) libraries of day 21 fibroblast, naive and $\mathrm{TSC}^{\mathrm{d} 8}$ reprogramming intermediates. b, Strength of EPI signatures on FDL (10,518 cells). The cell population not enriched for EPI signatures but enriched for TE signatures is indicated by a purple arrow, related to Fig. 4b. c, Representation of 13 cell clusters from unsupervised clustering projected onto the FDL, fibroblast medium cell clusters: D21fm1-D21fm7; naive reprogramming cell clusters: D21nr1-D21nr3; trophoblast reprogramming cell clusters: D21tr1-D21tr3, and d, Contribution of each scRNA-seq library (\%) to the composition of cell clusters. D21tr1 cluster is indicated by a purple arrow. e, Expression of genes associated with human fibroblasts (ANPEP), shared pluripotency (NANOG), primed pluripotency (ZIC2), naive pluripotency (DNMT3L) and trophoblast (GATA3) on FDL projection of day 21 fibroblast, naive and $\mathrm{TSC}^{\mathrm{d} 8}$ reprogramming intermediates scRNA-seq libraries (upper panels). Defined fibroblast, early-primed, primed, novel-intermediate and naive signatures (Extended Data Fig. 2f) on the FDL projection (bottom panels). f, Experimental designs to validate the potential of day 21 fibroblast reprogramming intermediates for the derivation of primed, naive hiPSCs and iTSCs. g, Phase-contrast images of primed, naive hiPSCs and iTSCs 
1596 generated from day 21 fibroblast reprogramming intermediates, $n=2$. Scale bar, $50 \mu \mathrm{m}$.

1597

1598

1599

1600

1601

1602

1603

1604

1605

1606

1607

1608

1609

1610

1611

1612

1613

1614

1615

1616

1617

1618

1619

1620

1621

1622

1623

1624

1625

1626

1627

Immunostaining of primed, naive hiPSCs and iTSCs with NANOG, KLF17, NR2F2, KRT7 and DAPI for nuclei staining, $n=2$. Scale bar, $200 \mu \mathrm{m}$. h, qRT-PCR analysis of NANOG, ZIC2, KLF17, DPPA3, GATA2, KRT7 expression in primed, naive hiPSCs and iTSCs generated from day 21 fibroblast reprogramming intermediates, $n=3$. Data are represented as mean \pm s.e.m. For more details on sample number and statistics, please see statistics and reproducibility section.

Extended Data Fig. 10 | Characterisation of iTSC ${ }^{\text {d8 }}$. a, Sendai viral transgenes in iTSC lines with positive and negative controls, $n=6$. b, Gene expression of trophoblast genes in fibroblasts, primed hiPSCs, naive t2iLGoY hiPSCs, iTSC ${ }^{\mathrm{d} 8}$ and $\mathrm{iTSC}^{\mathrm{d} 2 \mathrm{ln}}$ compared to TSCs derived from a human blastocyst (TSC ${ }^{\text {blast }}$ ) and first-trimester placental trophoblast $\left(\mathrm{TSC}^{\mathrm{CT}}\right)^{7}$, data are presented as mean $(n=2)$. c, Cell fusion index of $\mathrm{iTSC}^{\mathrm{d} 8}-\mathrm{ST}$ and iTSC ${ }^{\mathrm{d} 8}, n=5$, data are represented as mean \pm s.e.m., $p$ values by two-tailed unpaired Student's $t$-test. d, Representative results for hCG pregnancy test obtained from media of ST cells differentiated from $\mathrm{iTSC}^{\mathrm{d} 8}, n=6$. e, hCG levels of $\mathrm{iTSC}^{\mathrm{d} 8}$ and $\mathrm{iTSC}^{\mathrm{d} 8}-\mathrm{ST}$ conditioned media detected by ELISA , $n=4$. f, Representative flow cytometry analysis of pan HLA-A, B, C class I marker (W6/32), HLA-Bw4 and HLA-G in fibroblasts and EVTs, $n=4$. g, Representative flow cytometry analysis of pan HLA class I marker (W6/32) and HLA-G in iTSC ${ }^{\mathrm{d} 8}$-EVT and iTSC $^{\mathrm{d} 21 \mathrm{n}}$-EVT. h, Representative flow cytometry analysis of pan HLA class I marker (W6/32) in fibroblasts, primed hiPSCs, naive t2iLGoY hiPSCs, $\operatorname{iTSC}^{\mathrm{d} 8}$ and $\operatorname{iTSC}^{\mathrm{d} 21 \mathrm{n}}, n=4$. i, Expression of ST genes in iTSC ${ }^{\mathrm{d} 8}$ and $\mathrm{iTSC}^{\mathrm{d} 21 \mathrm{n}}$-derived ST cells and $\mathbf{j}$, expression of EVT genes in $\mathrm{iTSC}^{\mathrm{d} 8}$ and $\mathrm{iTSC}^{\mathrm{d} 21 \mathrm{n}}$-derived EVT cells. $\mathbf{k}$, Spearman correlation of the transcriptomes of fibroblast, primed and naive t2iLGoY hiPSCs, iTSC ${ }^{\mathrm{d} 8}$ and iTSC ${ }^{\mathrm{d} 21 \mathrm{n}}$, $i \mathrm{TSC}^{\mathrm{d} 8}-\mathrm{ST}$ and $\mathrm{iTSC}{ }^{\mathrm{d} 21 \mathrm{n}}-\mathrm{ST}, \mathrm{iTSC}{ }^{\mathrm{d} 8}-\mathrm{EVT}$ and $\mathrm{iTSC}^{\mathrm{d} 21 \mathrm{n}}-\mathrm{EVT}$ generated in this study with trophoblast organoids samples from Haider et al. ${ }^{29}$ and Turco et al. ${ }^{28}$ and $\mathbf{l}$, Single-cell fetalmaternal interface samples from Vento-Tormo et $\mathrm{al}^{27}, n \geq 2$, replicates are averaged prior to performing correlation. $\mathbf{m}$, Lesions harvested from subcutaneously engrafted $\mathrm{iTSC}^{\mathrm{d} 8}$ in NODSCID mice, $n=4$. For more details on sample number and statistics, please see statistics and reproducibility section.

\section{Acknowledgments}


1628 The authors thank the staff at Monash Flowcore Facility for providing high-quality cell

1629

1630

1631

1632

1633

1634

1635

1636

1637

1638

1639

1640

1641

1642

1643

1644

1645

1646

1647

1648

1649

1650

1651

1652

1653

1654

1655

1656

1657

1658

1659

1660

sorting service and technical input. The authors acknowledge the use of the services and facilities of Micromon, Monash Micro Imaging, and Monash Histology Platforms at Monash University. Furthermore, the authors thank Dr. Sen Wang and Dr. Trevor Wilson at the ACRF Centre for Cancer Genomic Medicine at the MHTP Medical Genomics Facility, and the University of Melbourne Centre for Cancer Research (UMCCR) core for assistance with next-generation library preparation and Illumina sequencing. We also thank Jess HatwellHumble for assistance with the mouse work. We thank A. Purcell (Monash University) for providing the HLA antibodies. This work was supported by National Health and Medical Research Council (NHMRC) project grants APP1104560 to J.M.Polo and A.L.L., APP1069830 to R.L., and a Monash University strategic grant awarded to C.M.N. X.L. was supported by the Monash International Postgraduate Research Scholarship, a Monash Graduate Scholarship and the Carmela and Carmelo Ridolfo Prize in Stem Cell Research. A.S.K. was supported by an NHMRC Early Career Fellowship APP1092280. J.M.Polo and R.L. were supported by Silvia and Charles Viertel Senior Medical Research Fellowships. J.M.Polo was also supported by an ARC Future Fellowship FT180100674. R.L was supported by a Howard Hughes Medical Institute International Research Scholarship. O.J.L.R. and J.F.O. were supported by a Singapore National Research Foundation Competitive Research Programme (NRF-CRP20-2017-0002). The Australian Regenerative Medicine Institute is supported by grants from the State Government of Victoria and the Australian Government.

\section{Author contributions}

J.M.Polo conceptualised the study. O.J.L.R. and J.M.Polo supervised the study. X.L., J.F.O., F.J.R., O.J.L.R. and J.M.Polo designed the experiments and analysis. O.J.L.R devised the single cells analysis pipeline and data integration. X.L. performed reprogramming experiments, collection and isolation of single cells, intermediates and functional validation of iTSC experiments with support from C.M.N., J.T., K.C.D., D.S.V., Y.B.Y.S., J.C., J.M.Paynter, J.F., Z.H., P.T., P.P.D., and S.K.N.; X.L. and C.M.N. performed single-cell RNA-seq, FACS experiments, SPADE analysis and the molecular experiments of the cells with support from A.S.K. and J.C.; L.G.M. helped with single nucleus-RNA-seq experiments with support from A.L.; M.R.L. helped with RT-PCR experiments. D.P. helped with sequencing of day 21 reprogramming intermediates scRNA-seq libraries. X.L. generated the lentiviral particles with the assistance of J.T., G.S.; J.P. helped with ATAC-seq experiments. 
1661 H.S.C., C.M.B., and A.L.L. provided reagents and technical assistance. H.N. and D.R.P 1662 helped with bulk RNA-seq analysis. J.F.O. performed the sn/scRNA-seq and bulk RNA-seq 1663 analyses for the human reprogramming intermediates and iTSC experiments as well as the 1664 integration across the various datasets with support from F.J.R., J.S., J.M.Polo and O.J.L.R.; 1665 F.J.R. performed ATAC-seq analysis with support from V.T., X.Y.C, J.S., S.B., O.J.L.R., 1666 W.A.P., D.C., A.T.C., J.M.Polo, and R.L.; J.F.O. and O.J.L.R. developed the interface for the 1667 interactive online tool. X.L., J.F.O., F.J.R., O.J.L.R., and J.M.Polo wrote the manuscript with 1668 input from K.C.D., A.G., A.T.C., L.D., C.M.N., and R.L. All authors approved of and 1669 contributed to, the final version of the manuscript.

\section{Competing interests}

1671 Although not directly related to this manuscript, O.J.L.R. and J.M.Polo. are co-inventors of 1672 the patent (WO/2017/106932) and are co-founders and shareholders of Mogrify Ltd., a cell therapy company. X.L., J.F.O., K.C.D., L.D., O.J.L.R. and J.M.Polo are co-inventors on a provisional patent application (application number: 2019904283) filed by Monash University, National University of Singapore and Université de Nantes related to work on derivation of iTSCs. The other authors declare no competing interests.

1677

1678 Additional information

1679 Supplementary information is available for this paper.

1680 Correspondence and requests for materials should be addressed to O.J.L.R. or J.M.Polo.

1681 Peer review information Nature thanks

1682 Reprints and permissions information is available at http://www.nature.com/reprints. 
a

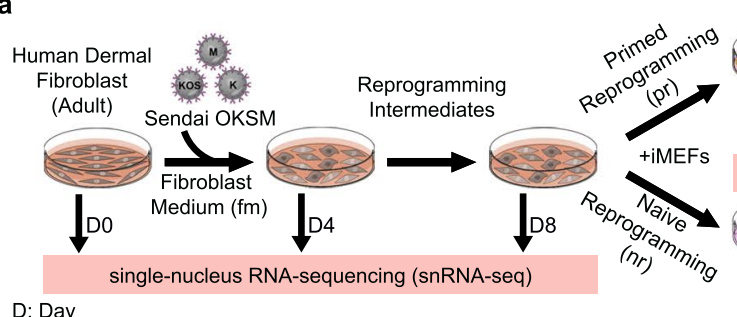
D: Day
P: Passage
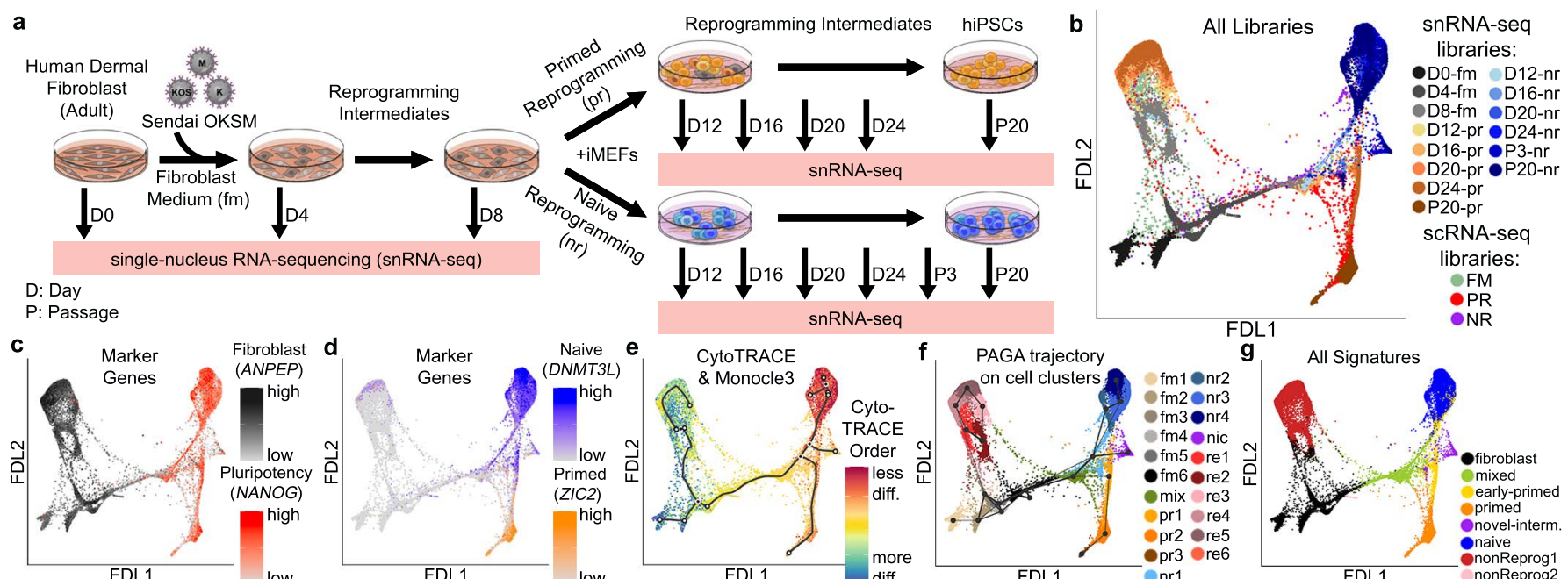

Reprogramming Intermediates hiPSCs

$\downarrow_{12} \downarrow_{116} \downarrow_{220} \downarrow_{224} \downarrow_{202}$

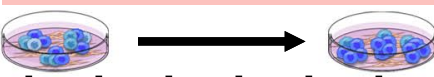

$\downarrow 12 \downarrow 16$

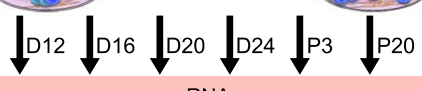

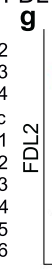

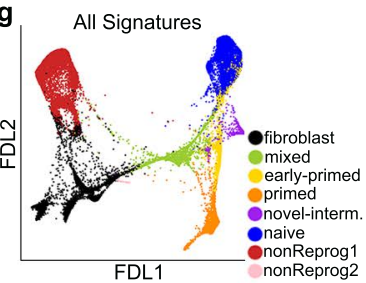


a

Integration of snRNA-seq (•)
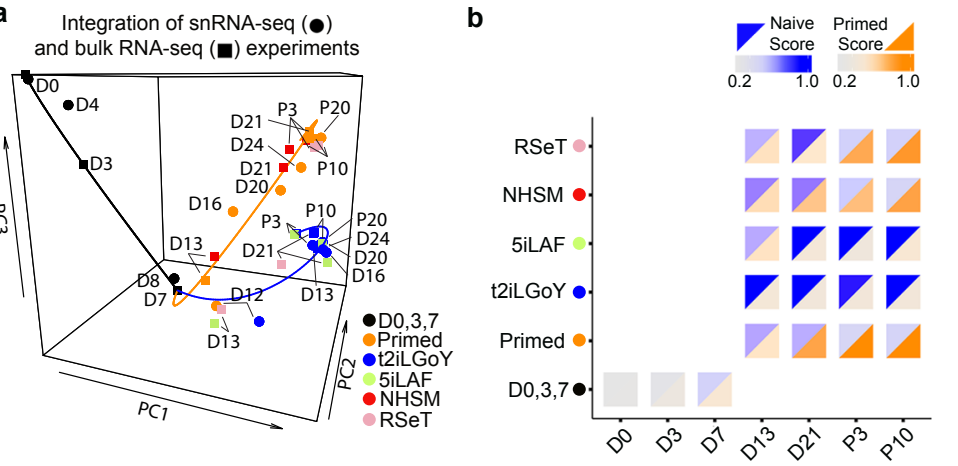

e

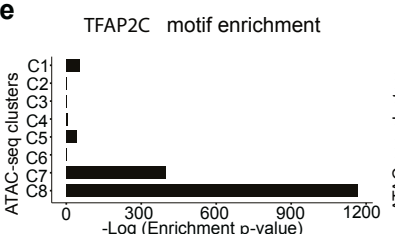

GATA2 motif enrichment

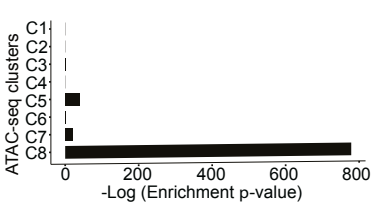

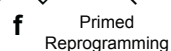

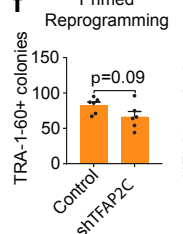

C ATAC-seq nucleosome-free signals

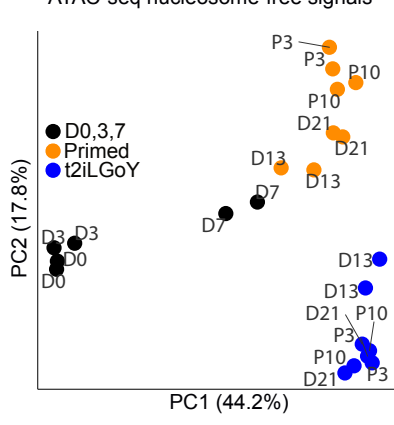

d Shared Loss (SL)

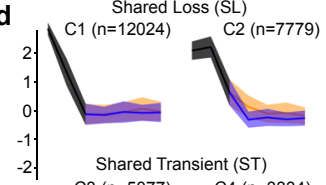

C3 ( $n=5077) \quad C 4(n=3334)$

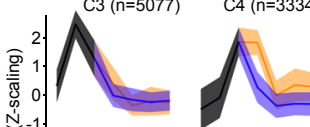

岕 0.1 .

永-2.

$\begin{array}{ll}\text { Shared Up (SU) } & \text { Primed Up (PU) } \\ \mathrm{C} 5(\mathrm{n}=9117) & \mathrm{C}=10129)\end{array}$

$\begin{array}{ccc}\text { Naive } & \text { Primed } & \text { Naive } \\ \text { Reprogramming of Reprogramming } & \text { Reprogramming }\end{array}$

Naive

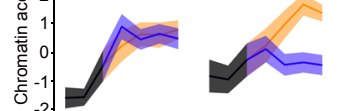

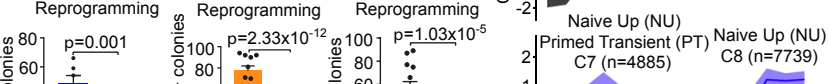

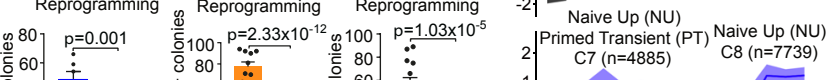

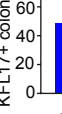

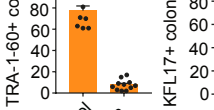

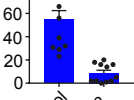

00

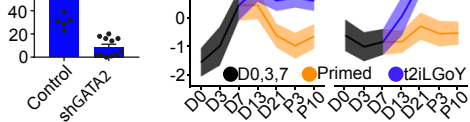

2. 0 OD,3,7 Primed Ot2iLGoY 


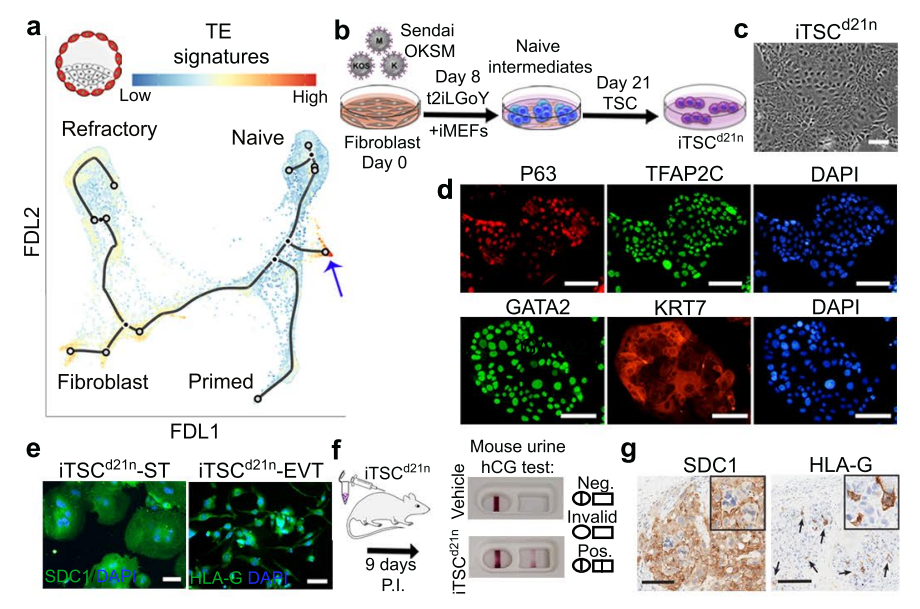




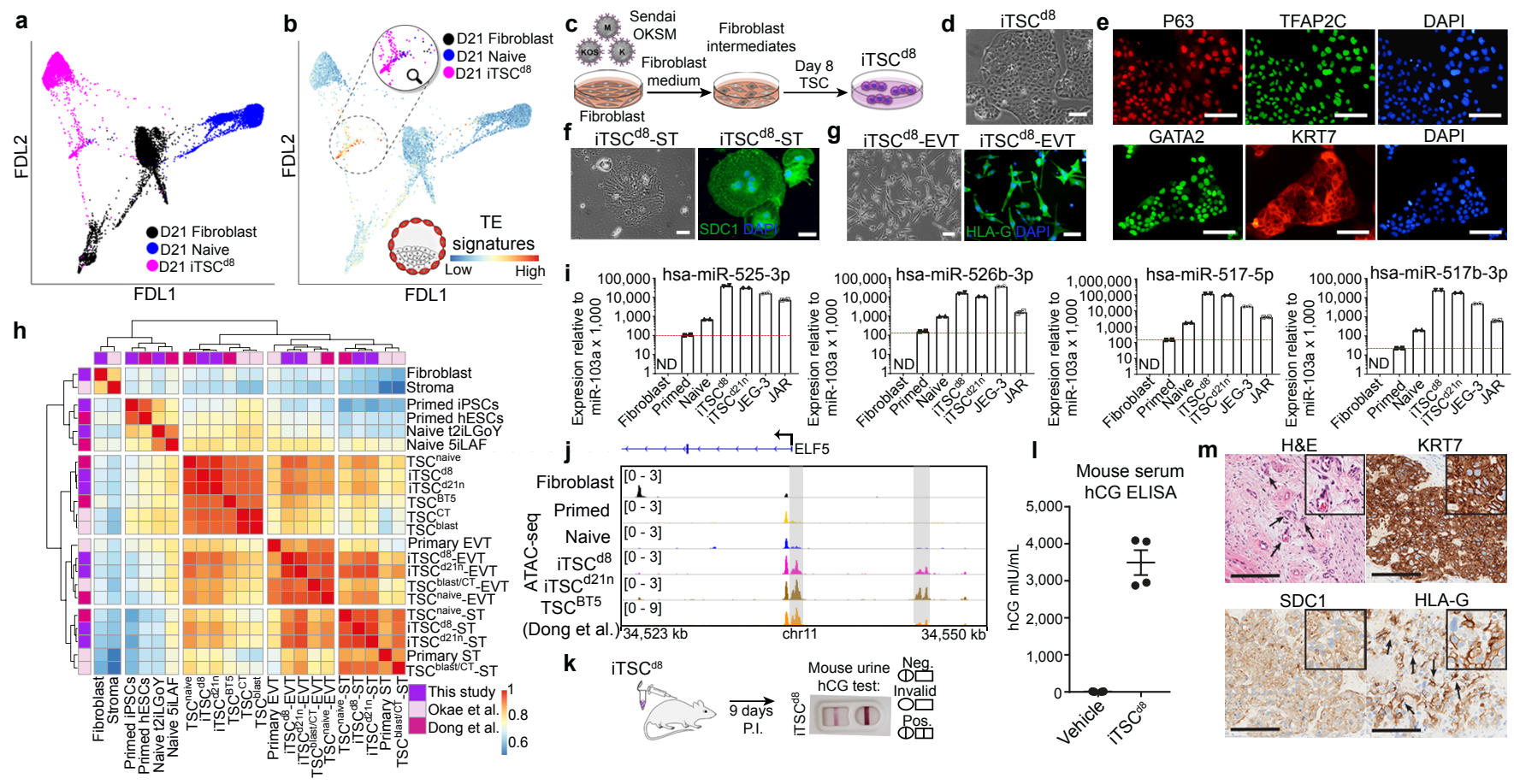

